\title{
OGLE-2005-BLG-071Lb, THE MOST MASSIVE M DWARF PLANETARY COMPANION?
}

Subo Dong ${ }^{1,2}$, Andrew Gould ${ }^{1,2}$, Andrzej Udalski ${ }^{3,4}$ JAY Anderson $^{5}$, G. W. Christie ${ }^{1,6}$, B. S. Gaudi ${ }^{1,2}$; And The OGLE Collaboration: M. Jaroszyński ${ }^{4}$, M. KubiaK ${ }^{4}$, M. K. Szymański ${ }^{4}$, G. PietrzyŃSki ${ }^{4}, 7$, I. SoszyŃSki ${ }^{4}$, O. SzewCZYK ${ }^{7,4}$, K. UlaczyK ${ }^{4}$, Ł. WyrzyKowski ${ }^{8,4}$; and The $\mu$ FUN Collaboration: D. L. DePoy ${ }^{2}$, D. B. Fox ${ }^{9}$, A. Gal-Yam ${ }^{10}$, C. HAN $^{11}$, S. LéPine ${ }^{12}$, J. McCormick ${ }^{13}$, E. OfFeK ${ }^{14}$, B.-G. PARK ${ }^{15}$, R. W. PogGE ${ }^{2}$; And The MOA Collaboration: F. Abe ${ }^{16}$,

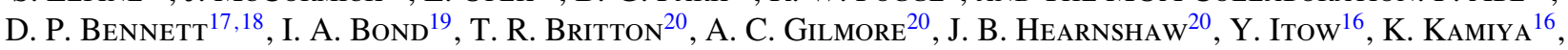

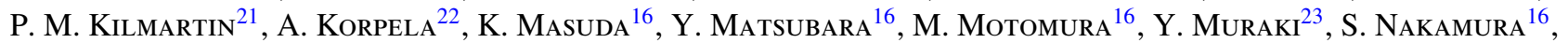
K. Ohnishi ${ }^{24}$, C. Okada ${ }^{16}$, N. Rattenbury ${ }^{25}$, To. Saito ${ }^{26}$, T. Sako ${ }^{16}$, M. Sasaki ${ }^{16}$, D. Sullivan ${ }^{22}$, T. Sumi ${ }^{16}$, P. J. Tristram ${ }^{21}$, T. Yanagisawa ${ }^{27}$, P. C. M. Yock $^{28}$, T. Yoshoika ${ }^{16}$; and The PlaneT/RoboNet Collaborations: M. D. Albrow ${ }^{20}$, J. P. Beaulieu ${ }^{29}$, S. Brillant ${ }^{30}$, H. Calitz ${ }^{31}$, A. Cassan ${ }^{32}$, K. H. CooK ${ }^{33}$, Ch. Coutures ${ }^{29}$, S. Dieters ${ }^{34}$,

D. Dominis Prester ${ }^{35}$, J. Donatowicz ${ }^{36}$, P. Fouqué ${ }^{37}$, J. Greenhill ${ }^{34}$, K. Hill ${ }^{34}$, M. Hoffman ${ }^{31}$, K. Horne ${ }^{38}$, U. G. Jørgensen ${ }^{39}$, S. Kane ${ }^{40}$, D. Kubas ${ }^{30}$, J. B. Marquette ${ }^{29}$, R. Martin ${ }^{41}$, P. Meintjes ${ }^{31}$, J. Menzies ${ }^{42}$, K. R. Pollard ${ }^{20}$, K. C. SAhu ${ }^{5}$, C. Vinter ${ }^{39}$, J. Wambsganss ${ }^{32}$, A. Williams ${ }^{41}$, M. Bode ${ }^{43}$, D. M. Bramich ${ }^{44}$, M. Burgdorf ${ }^{43}$, C. Snodgrass ${ }^{30}$, I. Steele $^{43}$; Vanessa Doublier ${ }^{45}$, and Cedric Foellmi ${ }^{46}$

${ }^{1}$ Microlensing Follow-Up Network ( $\mu$ FUN)

${ }^{2}$ Department of Astronomy, Ohio State University, 140 W. 18th Ave., Columbus, OH 43210, USA; dong@ @astronomy.ohio-state.edu, gould@astronomy.ohio-state.edu, gaudi@astronomy.ohio-state.edu, depoy@astronomy.ohio-state.edu, pogge@astronomy.ohio-state.edu ${ }^{3}$ Optical Gravitational Lensing Experiment (OGLE)

${ }^{4}$ Warsaw University Observatory, Al. Ujazdowskie 4, 00-478 Warszawa, Poland; udalski@astrouw.edu.pl, mj@astrouw.edu.pl, msz@astrouw.edu.pl, mk@astrouw.edu.pl,pietrzyn@astrouw.edu.pl,soszynsk@astrouw.edu.pl,kulaczyk@astrouw.edu.pl

${ }^{5}$ Space Telescope Science Institute, Baltimore, MD, USA; jayander@stsci.edu

${ }^{6}$ Auckland Observatory, Auckland, New Zealand; gwchristie@ christie.org.nz

${ }^{7}$ Departamento de Fisica, Universidad de Concepción, Casilla 160-C, Concepción, Chile; szewczyk@astro-udec.cl

${ }^{8}$ Institute of Astronomy, University of Cambridge, Madingley Road, Cambridge, CB3 OHA, UK; wyrzykow @ ast.cam.ac.uk

${ }^{9}$ Astronomy \& Astrophysics, Pennsylvania State University, 525 Davey Laboratory, University Park, PA 16802, USA; dfox@astro.psu.edu

${ }^{10}$ Benoziyo Center for Astrophysics, Weizmann Institute of Science, 76100 Rehovot, Israel; avishay.gal-yam@ weizmann.ac.il

${ }^{11}$ Program of Brain Korea, Department of Physics, Chungbuk National University, 410 Seongbong-Rho, Hungduk-Gu, Chongju 371-763, Korea; cheongho@astroph.chungbuk.ac.kr

12 Department of Astrophysics, Division of Physical Sciences, American Museum of Natural History, Central Park West at 79th Street, New York, NY 10024, USA; lepine@amnh.org

${ }^{13}$ Farm Cove Observatory, Centre for Backyard Astrophysics, Pakuranga, Auckland New Zealand; farmcoveobs@xtra.co.nz

${ }^{14}$ Division of Physics, Mathematics and Astronomy, California Institute of Technology, Pasadena, CA 91125, USA; eran@ astro.caltech.edu

${ }^{15}$ Korea Astronomy and Space Science Institute, 61-1 Hwaam-Dong, Yuseong-Gu, Daejeon 305-348, Korea; bgpark@kasi.re.kr

${ }^{16}$ Solar-Terrestrial Environment Laboratory, Nagoya University, Nagoya, 464-8601, Japan

${ }^{17}$ Department of Physics, 225 Nieuwland Science Hall, Notre Dame University, Notre Dame, IN 46556, USA

${ }_{18}$ Probing Lensing Anomalies NETwork (PLANET) Collaboration

${ }^{19}$ Institute for Information and Mathematical Sciences, Massey University, Private Bag 102-904, Auckland 1330, New Zealand

${ }^{20}$ Department of Physics and Astronomy, University of Canterbury, Private Bag 4800, Christchurch 8020, New Zealand

${ }^{21}$ Mt. John Observatory, P. O. Box 56, Lake Tekapo 8770, New Zealand

${ }^{22}$ School of Chemical and Physical Sciences, Victoria University, Wellington, New Zealand

${ }^{23}$ Department of Physics, Konan University, Nishiokamoto 8-9-1, Kobe 658-8501, Japan

${ }^{24}$ Nagano National College of Technology, Nagano 381-8550, Japan

25 Jodrell Bank Centre for Astrophysics, The University of Manchester, Manchester, M13 9PL, UK

${ }^{26}$ Tokyo Metropolitan College of Industrial Technology, Tokyo 116-8523, Japan

${ }^{27}$ Advanced Space Technology Research Group, Institute of Aerospace Technology, Japan Aerospace Exploration Agency (JAXA), Tokyo, Japan

${ }^{28}$ Department of Physics, University of Auckland, Private Bag 92-019, Auckland 1001, New Zealand

${ }^{29}$ Institut d'Astrophysique de Paris, 98bis Boulevard Arago, 75014 Paris, France

${ }^{30}$ European Southern Observatory, Casilla 19001, Vitacura 19, Santiago, Chile

${ }^{31}$ Department of Physics/Boyden Observatory, University of the Free State, Bloemfontein 9300, South Africa

32 Astronomisches Rechen-Institut, Zentrum für Astronomie, Heidelberg University, Mönchhofstr. 12-14, 69120 Heidelberg, Germany

${ }^{33}$ Lawrence Livermore National Laboratory, IGPP, P. O. Box 808, Livermore, CA 94551, USA

${ }^{34}$ University of Tasmania, School of Maths and Physics, Private bag 37, GPO Hobart, Tasmania 7001, Australia

${ }^{35}$ Department of Physics, University of Rijeka, Omladinska 14, 51000 Rijeka, Croatia

${ }^{36}$ Department of Computing, Technical University of Vienna, Wiedner Hauptstrasse 10, Vienna, Austria

${ }^{37}$ LATT, Université de Toulouse, CNRS, 14 av. E. Belin, 31400 Toulouse, France

38 SUPA, University of St Andrews, School of Physics \& Astronomy, North Haugh, St. Andrews, KY16 9SS, UK

${ }^{39}$ Niels Bohr Institute, Astronomical Observatory, Juliane Maries Vej 30, DK-2100 Copenhagen, Denmark

${ }^{40}$ Michelson Science Center, California Institute of Technology, MS 100-22, 770 South Wilson Avenue, Pasadena, CA 91125, USA

${ }^{41}$ Perth Observatory, Walnut Road, Bickley, Perth 6076, Australia

${ }^{42}$ South African Astronomical Observatory, P. O. Box 9 Observatory 7935, South Africa

${ }^{43}$ Astrophysics Research Institute, Liverpool John Moores University, Twelve Quays House, Egerton Wharf, Birkenhead, CH41 1LD, UK

${ }^{44}$ Isaac Newton Group of Telescopes, Apartado de Correos 321, E-38700 Santa Cruz de la Palma, Canary Islands, Spain

${ }^{45}$ ESO, Karl-Schwarzschild-Strasse 2, D-85748 Garching bei Mnchen

${ }^{46}$ LAOG, Observatoire de Grenoble, BP 53 F-38041 Grenoble, France

Received 2008 April 9; accepted 2008 November 18; published 2009 April 6 


\section{ABSTRACT}

We combine all available information to constrain the nature of OGLE-2005-BLG-071Lb, the second planet discovered by microlensing and the first in a high-magnification event. These include photometric and astrometric measurements from the Hubble Space Telescope, as well as constraints from higher order effects extracted from the ground-based light curve, such as microlens parallax, planetary orbital motion, and finite-source effects. Our primary analysis leads to the conclusion that the host of Jovian planet OGLE-2005-BLG-071Lb is an M dwarf in the foreground disk with mass $M=0.46 \pm 0.04 M_{\odot}$, distance $D_{l}=3.2 \pm 0.4 \mathrm{kpc}$, and thick-disk kinematics $v_{\mathrm{LSR}} \sim 103 \mathrm{~km} \mathrm{~s}^{-1}$. From the best-fit model, the planet has mass $M_{p}=3.8 \pm 0.4 M_{\text {Jupiter }}$, lies at a projected separation $r_{\perp}=3.6 \pm 0.2 \mathrm{AU}$ from its host, and so has an equilibrium temperature of $T \sim 55 \mathrm{~K}$, that is, similar to Neptune. A degenerate model gives similar planetary mass $M_{p}=3.4 \pm 0.4 M_{\text {Jupiter }}$ with a smaller projected separation, $r_{\perp}=2.1 \pm 0.1 \mathrm{AU}$, and higher equilibrium temperature, $T \sim 71 \mathrm{~K}$. These results from the primary analysis suggest that OGLE-2005-BLG-071Lb is likely to be the most massive planet yet discovered that is hosted by an $\mathrm{M}$ dwarf. However, the formation of such high-mass planetary companions in the outer regions of $\mathrm{M}$ dwarf planetary systems is predicted to be unlikely within the core-accretion scenario. There are a number of caveats to this primary analysis, which assumes (based on real but limited evidence) that the unlensed light coincident with the source is actually due to the lens, that is, the planetary host. However, these caveats could mostly be resolved by a single astrometric measurement a few years after the event.

Key words: Galaxy: bulge - gravitational lensing - planetary systems

\section{INTRODUCTION}

Microlensing provides a powerful method to detect extrasolar planets. Although only six microlens planets have been found to date (Bond et al. 2004; Udalski et al. 2005; Beaulieu et al. 2006; Gould et al. 2006; Gaudi et al. 2008; Dong et al. 2008), these include two major discoveries. First, two of the planets are "cold Neptunes," a high discovery rate in this previously inaccessible region of parameter space, suggesting that this new class of extrasolar planets is common (Gould et al. 2006; Kubas et al. 2008). Second, the discovery of the first Jupiter/Saturn analog via a very high magnification event with substantial sensitivity to multiple planets indicates that solar system analogs may be prevalent among planetary systems (Gaudi et al. 2008). Recent improvements in search techniques and future major upgrades should increase the discovery rate of microlensing planets substantially (Gaudi 2008).

Routine analysis of planetary microlensing light curves yields the planet/star mass ratio $q$ and the planet-star projected separation $d$ (in units of the angular Einstein radius). However, because the lens-star mass $M$ cannot be simply extracted from the light curve, the planet mass $M_{p}=q M$ remains, in general, equally uncertain.

The problem of constraining the lens mass $M$ is an old one. When microlensing experiments were initiated in the early 1990s, it was generally assumed that individual mass measurements would be impossible and that only statistical estimates of the lens mass scale could be recovered. However, Gould (1992) pointed out that the mass and lens-source relative parallax, $\pi_{\text {rel }} \equiv \pi_{l}-\pi_{s}$, are simply related to two observable parameters, the angular Einstein radius, $\theta_{\mathrm{E}}$, and the Einstein radius projected onto the plane of the observer, $\tilde{r}_{\mathrm{E}}$,

$$
M=\frac{\theta_{\mathrm{E}}}{\kappa \pi_{\mathrm{E}}}, \quad \pi_{\text {rel }}=\theta_{\mathrm{E}} \pi_{\mathrm{E}} .
$$

Here, $\pi_{\mathrm{E}}=\mathrm{AU} / \tilde{r}_{\mathrm{E}}$ is the "microlens parallax" and $\kappa \equiv$ $4 G /\left(c^{2} \mathrm{AU}\right) \sim 8.1 \mathrm{mas} / M_{\odot}$. See Gould (2000b) for an illustrated derivation of these relations.

In principle, $\theta_{\mathrm{E}}$ can be measured by comparing some structure in the light curve to a "standard angular ruler" on the sky. The best example is light-curve distortions due to the finite angular radius of the source $\theta_{*}$ (Gould 1994), which usually can be estimated very well from its color and apparent magnitude (Yoo et al. 2004). While such finite-source effects are rare for microlensing events considered as a whole, they are quite common for planetary events. The reason is simply that the planetary distortions of the light curve are typically of a similar or smaller scale than $\theta_{*}$. In fact, all six planetary events discovered to date show such effects. Combining $\theta_{\mathrm{E}}$ with the (routinely measurable) Einstein radius crossing time $t_{\mathrm{E}}$ yields the relative proper motion $\mu$ in the geocentric frame,

$$
\mu_{\mathrm{geo}}=\frac{\theta_{\mathrm{E}}}{t_{\mathrm{E}}} .
$$

From Equation (1), measurement of $\theta_{\mathrm{E}}$ by itself fixes the product $M \pi_{\text {rel }}=\theta_{\mathrm{E}}^{2} / \kappa$. Using priors on the distribution of lens-source relative parallaxes, one can then make a statistical estimate of the lens mass $M$ and so of the planet mass $M_{p}$.

To do better, one must develop an additional constraint. This could be measurement of the microlens parallax $\pi_{\mathrm{E}}$, but this is typically possible only for long events. Another possibility is direct detection of the lens, either under the "glare" of the source during and immediately after the event or displaced from the source well after the event is over. Bennett et al. (2006) used the latter technique to constrain the mass of the first microlensing planet, OGLE-2003-BLG-235/MOA-2003-BLG53Lb. They obtained Hubble Space Telescope (HST) Advanced Camera for Surveys (ACS) images in $B, V$, and $I$ at an epoch $\Delta t=1.78$ years after the event. They found astrometric offsets of the (still overlapping) lens and source light among these images of up to 0.7 mas. Knowing the lens-source angular separation $\Delta \theta=\mu \Delta t$ from the already determined values of $\theta_{\mathrm{E}}$ and $t_{\mathrm{E}}$, they were able to use these centroid offsets to fix the color and magnitude of the lens and so (assuming that it was a main-sequence star) its mass.

While the planet mass is usually considered to be the most important parameter that is not routinely derivable from the light curve, the same degeneracy impacts two other quantities as well, the distance and the transverse velocity of the lens. Knowledge of these quantities could help constrain the nature of the lens, that is, whether it belongs to bulge, the foreground disk, or possibly the thick disk or even the stellar halo. Since microlensing is the only method currently capable of detecting planets in populations well beyond the solar neighborhood, extracting such 


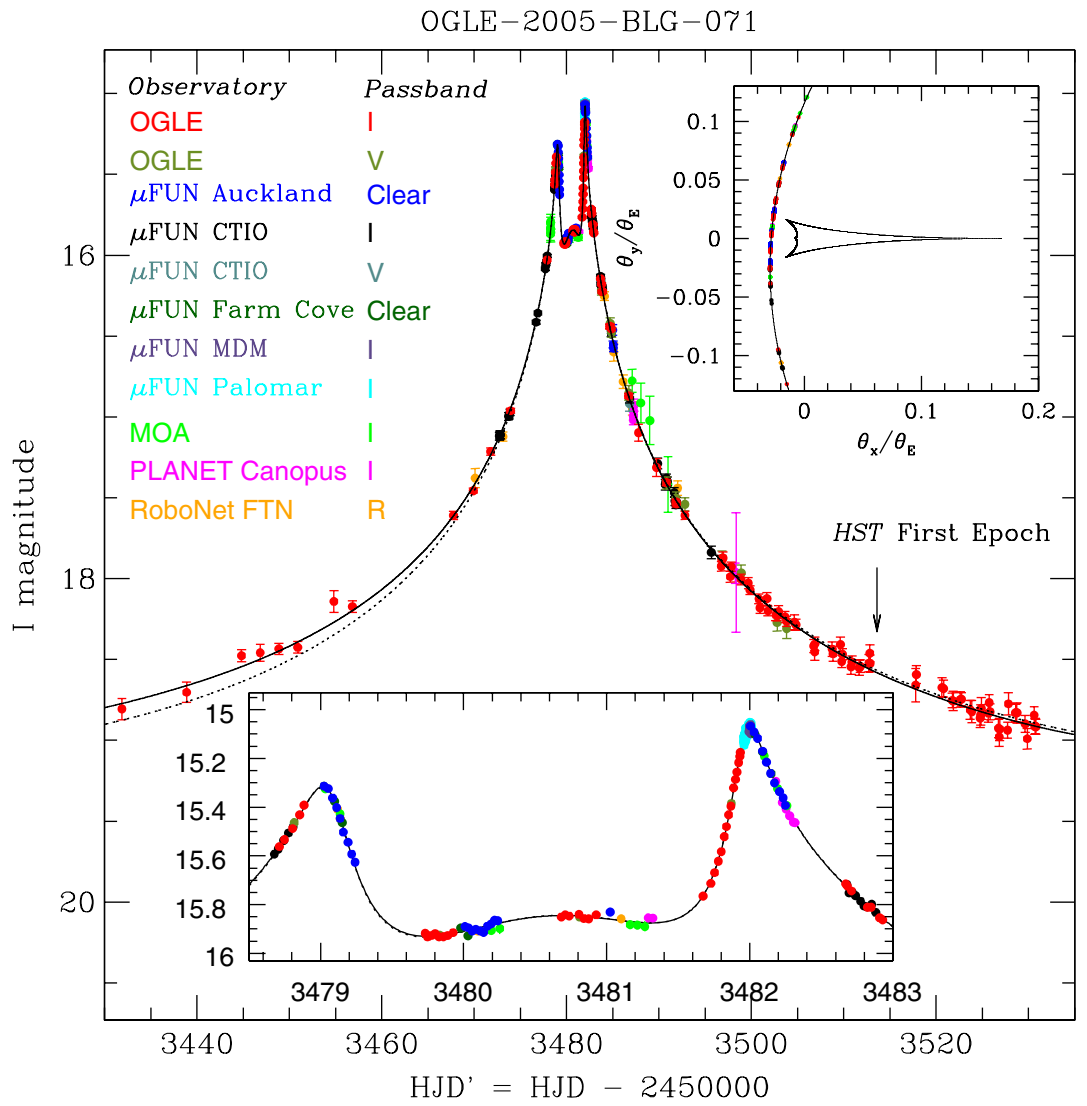

Figure 1. Main panel: all available ground-based data of the microlensing event OGLE-2005-BLG-071. HST ACS HRC observations in F814W and F555W were taken at two epochs, once when the source was magnified by $A \sim 2$ (arrow) and again at HJD $=2453788.2$ (at baseline). Planetary models that include (solid) and excludes (dotted) microlens parallax are shown. Zoom at bottom: triple-peak feature that reveals the presence of the planet. Each of the three peaks corresponds to the source passing by a cusp of the central caustic induced by the planet. Upper inset: trajectory of the source relative to the lens system in the units of angular Einstein radius $\theta_{\mathrm{E}}$. The lens star is at $(0,0)$, and the star-planet axis is parallel to the $x$-axis. The best-fit angular size of the source star in units of $\theta_{\mathrm{E}}$ is $\rho \sim 0.0006$, too small to be resolved in this figure.

information would be quite useful. Because the mass, distance, and transverse velocity are all affected by a common degeneracy, constraints on one quantity are simultaneously constraints on the others. As mentioned above, simultaneous measurements of $\theta_{\mathrm{E}}$ and $\pi_{\mathrm{E}}$ directly yield the mass. However, clearly from Equation (1), they also yield the distance and hence (from Equation (2)) also the transverse velocity. Here, we assemble all available data to constrain the mass, distance and transverse velocity of the second microlensing planet, OGLE-2005-BLG071Lb, whose discovery we previously reported (Udalski et al. 2005, hereafter Paper I).

\section{OVERVIEW OF DATA AND TYPES OF CONSTRAINTS}

The light curve consists of 1398 data points from nine groundbased observatories (see Figure 1), plus two epochs of HST ACS data in the F814W $(I)$ and F555W ( $V$ ) filters. The primary ground-based addition relative to Paper I is late-time data from OGLE, which continued to monitor the event down to baseline until HJD $=2453790.9$

These data potentially provide constraints on several parameters in addition to those reported in Paper I. First, the light curve shows a clear asymmetry between the rising and falling parts of the light curve, which is a natural result of microlens parallax due to the Earth's accelerated motion around the Sun (see the best-fit model without parallax effects plotted in a dotted line in Figure 1). However, it is important to keep in mind that such distortions are equally well produced by "xallarap" due to accelerated motion of the source around a companion. Poindexter et al. (2005) showed that it can be difficult to distinguish between the two when, as in the present case, the effect is detected at $\Delta \chi^{2} \lesssim 100$.

Second, the two pronounced peaks of the light curve, which are due to "cusp approaches" (see the bottom inset of Figure 1), are relatively sharp and have good coverage. These peaks would tend to be "rounded out" by finite-source effects, so in principle it may be possible to measure $\rho$ (i.e., $\theta_{*}$ in the units of $\theta_{\mathrm{E}}$ ) from these distortions.

Third, the orbital motion of the planet can give rise to two effects: rotation of the caustic about the center of the mass and distortion of the caustic due to expansion/contraction of the planet-star axis. The first changes the orientation of the caustic structure as the event evolves while the second changes its shape. These effects are expected to be quite subtle because the orbital period is expected to be of an order of 10 years while the source probes the caustic structure for only about four days. Nevertheless, they can be very important for the interpretation of the event.

Finally, the HST data cover two epochs, one at 2005 May 23 (indicated by the arrow in Figure 1) when the magnification was about $A=2$ and the other at 2006 February 21 when the event was very nearly at baseline, $A \sim 1$. These data could potentially yield four types of information. First, they can effectively determine whether the blended light is "associated" with the event or not. The blended light is composed of sources in the same photometric aperture as the magnified source, but 
Table 1

Light-Curve Parameter Estimations from MCMC Simulations

\begin{tabular}{|c|c|c|c|c|c|c|c|c|c|c|c|c|c|c|c|}
\hline $\begin{array}{l}\text { Model } \\
\chi^{2} \\
\end{array}$ & $\begin{array}{c}t_{0} \\
\text { (HJD') } \\
\end{array}$ & $u_{0}$ & $\begin{array}{c}t_{\mathrm{E}} \\
\text { (day) }\end{array}$ & $d$ & $\begin{array}{c}q \\
\times 10^{3} \\
\end{array}$ & $\begin{array}{c}\alpha \\
(\mathrm{deg}) \\
\end{array}$ & $\begin{array}{c}\rho \\
\times 10^{4} \\
\end{array}$ & N & $\pi_{\mathrm{E}, E}$ & $\begin{array}{c}\omega \\
\left(\mathrm{yr}^{-1}\right) \\
\end{array}$ & $\begin{array}{c}\dot{d} / d \\
\left(\mathrm{yr}^{-1}\right) \\
\end{array}$ & $\begin{array}{c}I_{\mathrm{s}} \\
(\mathrm{mag})\end{array}$ & $\begin{array}{c}I_{\mathrm{b}} \\
(\mathrm{mag})\end{array}$ & $\begin{array}{c}V_{\mathrm{s}} \\
(\mathrm{mag})\end{array}$ & $\begin{array}{c}V_{\mathrm{b}} \\
(\mathrm{mag}) \\
\end{array}$ \\
\hline \multicolumn{16}{|c|}{ MCMC } \\
\hline $\begin{array}{l}\text { Wide+ } \\
1345.0 \\
\end{array}$ & $\begin{array}{r}3480.7 \\
+0.00 \\
-0.00 \\
\end{array}$ & & $\begin{array}{l}71.1 \\
+2.3 \\
-2.4 \\
\end{array}$ & & $\begin{array}{c}7.5 \\
\pm 0.2 \\
\end{array}$ & & $\begin{array}{r}3.9 \\
+1.8 \\
-2.7 \\
\end{array}$ & & & & & & & $\begin{array}{l}20.85 \\
\pm 0.04 \\
\end{array}$ & $\begin{array}{l}23.11 \\
+0.43 \\
-0.26 \\
\end{array}$ \\
\hline $\begin{array}{l}\text { Wide- } \\
1345.3 \\
\end{array}$ & $\begin{array}{r}3480.7 \\
+0.00 \\
-0.00 \\
\end{array}$ & & & & $\begin{array}{c}7.5 \\
\pm 0.3 \\
\end{array}$ & & $\begin{array}{r}3.9 \\
+1.8 \\
-2.6 \\
\end{array}$ & & & & & & & & \\
\hline $\begin{array}{l}\text { Close+ } \\
1345.8 \\
\end{array}$ & $\begin{array}{c}3480.6789 \\
+0.0055 \\
-0.0043 \\
\end{array}$ & & $\begin{array}{l}70.1 \\
+2.1 \\
-2.4 \\
\end{array}$ & & $\begin{array}{c}6.9 \\
\pm 0.3 \\
\end{array}$ & 27427 & $\begin{array}{r}3.1 \\
+1.7 \\
-2.5 \\
\end{array}$ & & & & & & & & \\
\hline $\begin{array}{l}\text { lose- } \\
345.2\end{array}$ & $\begin{array}{c}3480.6799 \\
+0.0042 \\
-0.0051 \\
\end{array}$ & & $\begin{array}{l}69.2 \\
+2.3\end{array}$ & & $\begin{array}{c}6.9 \\
\pm 0.3 \\
\end{array}$ & & $\begin{array}{c}2.7 \\
\pm 2.2 \\
\end{array}$ & & & & & $\begin{array}{l}19.52 \\
\pm 0.03 \\
\end{array}$ & $\begin{array}{l}21.30 \\
\pm 0.18 \\
\end{array}$ & $\begin{array}{l}20.85 \\
\pm 0.04 \\
\end{array}$ & $\begin{array}{l}23.17 \\
+0.35 \\
-0.32 \\
\end{array}$ \\
\hline \multicolumn{16}{|c|}{ MCMC B } \\
\hline 1353.4 & $\begin{array}{r}3480 \\
+0.0 \\
-0.0 \\
\end{array}$ & $\begin{array}{c}0.0287 \\
\pm 0.0007 \\
\end{array}$ & ${ }_{-1}^{+1}$ & & $\begin{array}{c}7.7 \\
\pm 0.2 \\
\end{array}$ & $\begin{array}{l}+0.17 \\
-0.11 \\
\end{array}$ & $\begin{array}{c}6.1 \\
\pm 0.4 \\
\end{array}$ & & & $\begin{array}{l}-1.242 \\
+0.321 \\
-0.125 \\
\end{array}$ & $\begin{array}{l}-0.283 \\
\pm 0.129 \\
\end{array}$ & $\begin{array}{l}19.49 \\
\pm 0.03 \\
\end{array}$ & $\begin{array}{l}21.40 \\
\pm 0.19 \\
\end{array}$ & $\begin{array}{l}20.82 \\
\pm 0.03 \\
\end{array}$ & $\begin{array}{l}23.91 \\
+0.24 \\
-0.20 \\
\end{array}$ \\
\hline $\begin{array}{l}\text { Wide- } \\
1353.3 \\
\end{array}$ & $\begin{array}{c}3480.7012 \\
+0.0052 \\
-0.0060 \\
\end{array}$ & $\begin{array}{l}-0.0287 \\
\pm 0.0007 \\
\end{array}$ & $\begin{array}{l}69.2 \\
+1.7 \\
-1.8 \\
\end{array}$ & $\begin{array}{l}1.305 \\
+0.002 \\
-0.005 \\
\end{array}$ & $\begin{array}{c}7.7 \\
\pm 0.2 \\
\end{array}$ & $\begin{array}{l}86.29 \\
+0.12 \\
-0.15 \\
\end{array}$ & $\begin{array}{r}6.0 \\
+0.5 \\
-0.3 \\
\end{array}$ & $\begin{array}{r}0.02 \\
+0.10 \\
-0.13 \\
\end{array}$ & $\begin{array}{c}-0.21 \\
\pm 0.03 \\
\end{array}$ & $\begin{array}{l}+0.127 \\
-0.342\end{array}$ & $\begin{array}{l}-0.293 \\
+0.131 \\
-0.121 \\
\end{array}$ & $\begin{array}{l}19.49 \\
\pm 0.02 \\
\end{array}$ & $\begin{array}{l}21.40 \\
\pm 0.19 \\
\end{array}$ & $\begin{array}{c}20.82 \\
\pm 0.03 \\
\end{array}$ & $\begin{array}{l}23.97 \\
+0.19 \\
-0.24 \\
\end{array}$ \\
\hline $\begin{array}{l}\text { Close+ } \\
1355.5 \\
\end{array}$ & $\begin{array}{c}3480.6792 \\
+0.0041 \\
-0.0051 \\
\end{array}$ & 0245 & $\begin{array}{l}68.3 \\
\pm 1.6 \\
\end{array}$ & $\begin{array}{l}0.763 \\
+0.003 \\
-0.006 \\
\end{array}$ & $\begin{array}{c}7.0 \\
+0.3 \\
-0.2 \\
\end{array}$ & $\begin{array}{c}274.38 \\
+0.23 \\
-0.39 \\
\end{array}$ & $\begin{array}{c}6.0 \\
\pm 0.4 \\
\end{array}$ & $\begin{array}{l}-0.01 \\
+0.12 \\
-0.15 \\
\end{array}$ & $\begin{array}{c}-0.22 \\
\pm 0.02 \\
\end{array}$ & $\begin{array}{l}0.415 \\
+0.503 \\
-0.744 \\
\end{array}$ & $\begin{array}{l}0.569 \\
+0.112 \\
-0.130 \\
\end{array}$ & $\begin{array}{l}19.49 \\
\pm 0.03 \\
\end{array}$ & $\begin{array}{l}21.35 \\
+0.20 \\
-0.16 \\
\end{array}$ & $\begin{array}{l}20.83 \\
\pm 0.02 \\
\end{array}$ & $\begin{array}{l}23.88 \\
+0.24 \\
-0.18 \\
\end{array}$ \\
\hline $\begin{array}{l}\text { Close- } \\
1355.5\end{array}$ & $\begin{array}{c}3480.6793 \\
+0.0042 \\
-0.0051\end{array}$ & $\begin{array}{l}-0.0245 \\
\pm 0.0006\end{array}$ & $\begin{array}{l}68.2 \\
+1.8 \\
-1.5\end{array}$ & $\begin{array}{l}0.762 \\
+0.004 \\
-0.006\end{array}$ & $\begin{array}{c}7.1 \\
\pm 0.3\end{array}$ & $\begin{array}{l}85.63 \\
+0.46 \\
-0.24\end{array}$ & $\begin{array}{c}6.0 \\
\pm 0.4\end{array}$ & $\begin{array}{c}0.04 \\
+0.09 \\
-0.16\end{array}$ & $\begin{array}{c}-0.22 \\
\pm 0.03\end{array}$ & $\begin{array}{l}-0.179 \\
+0.703 \\
-0.722\end{array}$ & $\begin{array}{l}0.561 \\
+0.126 \\
-0.112\end{array}$ & $\begin{array}{l}19.50 \\
\pm 0.02\end{array}$ & $\begin{array}{l}21.36 \\
\pm 0.18\end{array}$ & $\begin{array}{l}20.83 \\
\pm 0.02\end{array}$ & $\begin{array}{l}23.90 \\
\pm 0.21\end{array}$ \\
\hline
\end{tabular}

that do not become magnified during the event. If this light is due to the lens, a companion to the lens, or a companion to the source, it should fall well within the ACS point-spread function (PSF) of the source. On the other hand, if it is due to a random interloper along the line of sight (LOS), then it should be separately resolved by the ACS or at least give rise to a distorted PSF. Second, the $H S T$ data can greatly improve the estimate of the color of the blended light. The original model determined the source fluxes in both OGLE $V$ and $I$ very well, and of course the baseline fluxes are also quite well determined. So it would seem that the blended fluxes, which are the differences between these two, would also be well determined. This proves to be the case in the $I$ band. However, while the source flux is derivable solely from flux differences over the light curve (and so is well determined from OGLE difference image analysis (DIA); Wozniak 2000), the baseline flux depends critically on the zero point of PSF-fitting photometry, whose accuracy is fundamentally limited in very crowded bulge fields. The small zero-point errors turn out to have no practical impact for the relatively bright $I$ background light, but are important for the $V$ band. Third, one might hope to measure a centroid shift between the two colors in the manner of Bennett et al. (2006). Last, one can derive the source proper motion $\mu_{s}$ from $H S T$ data (at least relative to the mean motion of bulge stars). This is important, because the event itself yields the source-lens relative proper motion, $\boldsymbol{\mu}_{\mathrm{geo}}$. Hence, precise determination of $\boldsymbol{\mu}_{l}$ requires knowledge of two proper-motion differences, first the heliocentric proper motion:

$$
\mu_{\mathrm{hel}}=\mu_{l}-\mu_{s},
$$

and second, the offset between the heliocentric and geocentric proper motions:

$$
\boldsymbol{\mu}_{\mathrm{hel}}-\boldsymbol{\mu}_{\mathrm{geo}}=\frac{\boldsymbol{v}_{\oplus} \pi_{\mathrm{rel}}}{\mathrm{AU}} .
$$

Here, $\boldsymbol{v}_{\oplus}$ is the velocity of the Earth relative to the Sun at the time of peak magnification $t_{0}$. Note that if the lens-source relative parallax $\pi_{\text {rel }}$ is known, even approximately, then the latter difference can be determined quite well, since its total magnitude is just $0.6 \mathrm{mas} \mathrm{yr}^{-1}\left(\pi_{\mathrm{rel}} / 0.17 \mathrm{mas}\right)$.

\section{CONSTRAINING THE PHYSICAL PROPERTIES OF THE LENS AND ITS PLANETARY COMPANION}

In principle, all the effects summarized in Section 2 could interact with each other and with the parameters previously determined, leading potentially to a very complex analysis. In fact, we will show that most effects can be treated as isolated from one another, which greatly facilitates the exposition. In the following sections, we will discuss the higher order microlens effects in the order of their impact on the ground-based light curve, starting with the strongest, that is, parallax effects (Section 3.1), followed by planetary orbital motion (Section 3.2) and finally the weakest, finite-source effects (Section 3.3.4). To study these effects, we implement Markov chain Monte Carlo (MCMC) with an adaptive step-size Gaussian sampler (Doran \& Mueller 2003) to perform the model fitting and obtain the uncertainties of the parameters. The HST astrometry is consistent with no $(V-I)$ color-dependent centroid shift in the first epoch, while such a shift is seen in the second epoch observations (Section 3.4). In addition, the PSF of the source shows no sign of broadening due to the blend, suggesting that the blend is associated with the event (Section 3.5). Therefore, the $H S T$ observations provide good evidence that the blend is likely due to the lens. In Section 3.4, it is shown, under such an assumption, how the astrometry can be used in conjunction with finite-source and microlens parallax measurements to constrain the angular Einstein radius and proper motion (Section 3.4). In Section 3.5, we discuss using HST photometric constraints in the form of $\chi^{2}$ penalties to the MCMC runs to extract the color and brightness of the blend. The results of these runs, which include all higher order effects of the ground-based light curve and the $H S T$ photometric constraints, are summarized as "MCMC A" in Table 1. Subsequently in Section 3.6, by making the assumption that the blended light seen by HST is due to the lens, we combine 
Table 2

Derived Physical Parameters

\begin{tabular}{lcccccccc}
\hline \hline $\begin{array}{l}\text { Model } \\
\chi^{2}\end{array}$ & $\begin{array}{c}M \\
M_{\odot}\end{array}$ & $\begin{array}{c}\pi_{\mathrm{rel}} \\
\text { mas }\end{array}$ & $\begin{array}{c}D_{l} \\
\mathrm{kpc}\end{array}$ & $\begin{array}{c}\mu_{\mathrm{N}} \\
\text { mas yr }^{-1}\end{array}$ & $\begin{array}{c}\mu_{\mathrm{E}} \\
\mathrm{mas} \mathrm{yr}^{-1}\end{array}$ & $\begin{array}{c}\theta_{\mathrm{E}} \\
\text { mas }\end{array}$ & $\begin{array}{c}M_{p} \\
M_{\mathrm{Jupiter}}\end{array}$ & $\begin{array}{c}r_{\perp} \\
\mathrm{AU}\end{array}$ \\
\hline Wide+ & 0.46 & 0.19 & 3.2 & -0.4 & -4.3 & 0.84 & 3.8 & 3.6 \\
1353.4 & \pm 0.04 & \pm 0.04 & \pm 0.4 & +2.7 & \pm 0.3 & +0.06 & +0.3 & \pm 0.2 \\
\hline Wide- & 0.46 & 0.19 & 3.2 & 0.3 & -4.3 & 0.85 & 3.8 & 3.6 \\
1353.3 & \pm 0.04 & +0.04 & \pm 0.4 & +2.3 & +0.3 & \pm 0.05 & ${ }_{-0.3}$ & \pm 0.2 \\
\hline Close+ & 0.46 & 0.19 & 3.1 & -2.6 & -4.4 & 0.86 & 3.4 & 2.1 \\
1355.5 & \pm 0.04 & +0.04 & \pm 0.4 & +4.8 & \pm 0.3 & \pm 0.05 & ${ }_{-0.3}$ & \pm 0.1 \\
\hline Close- & 0.46 & 0.20 & 3.1 & -0.2 & -4.4 & 0.87 & 3.4 & 2.1 \\
1355.5 & \pm 0.04 & \pm 0.04 & \pm 0.3 & +3.6 & \pm 0.3 & \pm 0.04 & \pm 0.3 & \pm 0.1 \\
\hline
\end{tabular}

all constraints discussed above to obtain physical parameters of the lens star and its planet. The corresponding best-fit model parameters are reported as "MCMC B" in Table 1. The results for the physical parameters from these runs are given in Table 2. Finally, we discuss some caveats in the analysis in Sections 3.7 and 3.8 .

\subsection{Microlens Parallax Effects}

A point-source static binary-lens model has six "geometricmodel" parameters: three "single-lens" parameters $\left(t_{0}, u_{0}, t_{\mathrm{E}}\right)$, where we define the time of "peak" magnification (actually lens-source closest approach) $t_{0}$ and the impact parameter $u_{0}$ with respect to the center of mass of the planet-star systems, and three "binary-lens" parameters $(q, d, \alpha)$, where $\alpha$ is the angle between the star-planet axis and the trajectory of the source relative to the lens. In addition, flux parameters are included to account for light coming from the source star $\left(F_{s}\right)$ and the blend $\left(F_{b}\right)$ for each data set. In this paper, we extend the fitting by including microlens parallax, orbital motion, and finite-source effects. Paper I reported that, within the context of the point-source static binary-lens models, the best-fit widebinary $(d>1)$ solution is preferred by $\Delta \chi^{2}=22$ over the closebinary $(d<1)$ solution. Remarkably, when we take account of parallax, finite-source, and orbital effects, this advantage is no longer as significant. We discuss the wide/close degeneracy with more detail in Section 3.6.2.

The microlens parallax effects are parametrized by $\pi_{\mathrm{E}, \mathrm{E}}$ and $\pi_{\mathrm{E}, \mathrm{N}}$, following the geocentric parallax formalism by An et al. (2002) and Gould (2004). To properly model the parallax effects, we characterize the "constant acceleration degeneracy" (Smith et al. 2003) by probing models with $u_{0} \rightarrow-u_{0}$ and $\alpha \rightarrow-\alpha$. We find that all other parameters remain essentially unchanged under this form of degeneracy. In the following sections, if not otherwise specified, parameters from models with positive $u_{0}$ are adopted.

As shown in Figure 2, microlens parallax is firmly detected in this event at a greater than $8 \sigma$ level. Not surprisingly, the error ellipse of $\pi_{\mathrm{E}}$ is elongated toward $\pi_{\mathrm{E}, \perp}$, that is, the direction perpendicular to the position of the Sun at the peak of event, projected onto the plane of the sky (Gould et al. 1994; Poindexter et al. 2005). As a result, $\pi_{\mathrm{E}, \mathrm{E}}$ is much better determined than $\pi_{\mathrm{E}, \mathrm{N}}$,

$$
\begin{aligned}
& \pi_{\mathrm{E}, \mathrm{E}}=-0.26 \pm 0.05, \quad \pi_{\mathrm{E}, \mathrm{N}}=-0.30_{-0.28}^{+0.24} \quad \text { (wide), } \\
& \pi_{\mathrm{E}, \mathrm{E}}=-0.27 \pm 0.05, \quad \pi_{\mathrm{E}, \mathrm{N}}=-0.36_{-0.27}^{+0.24} \quad \text { (close). }
\end{aligned}
$$

Xallarap (light-curve distortion from reflex motion of the source due to a binary companion) could provide an alternate

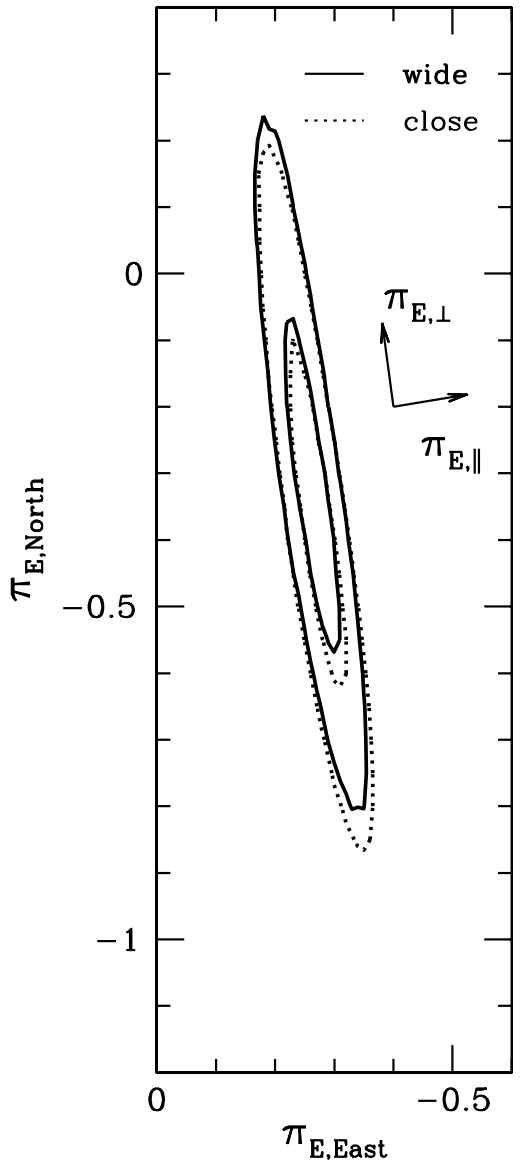

Figure 2. Probability contours $\left(\Delta \chi^{2}=1,4\right)$ of microlens parallax parameters derived from MCMC simulations for wide-binary (in a solid line) and closebinary (in a dashed line) solutions. Figure 2 and Equation (12) in Gould (2004) imply that $\pi_{E, \perp}$ is defined so that $\pi_{E, \|}$ and $\pi_{E, \perp}$ form a right-handed coordinate system.

explanation of the detected parallax signals. In Section 3.8, we find that the best-fit xallarap parameters are consistent with those derived from the Earth's orbit, a result that favors the parallax interpretation.

\subsection{Fitting Planetary Orbital Motion}

To model orbital motion, we adopt the simplest possible model, with a uniform expansion rate $\dot{b}$ in binary separation $b$ and uniform binary rotation rate $\omega$. Because orbital effects are operative only for about four days, while the orbital period is of an order of 10 years, this is certainly adequate. Interestingly, the orbital motion is more strongly detected for the close solutions (at a $\gtrsim 5.5 \sigma$ level) than the wide solutions (at a $\sim 3 \sigma$ level), and as a result, it significantly lessens the previous preference of the wide solution that was found before orbital motion was taken into account. Further discussions on planetary orbital motion are given in Section 3.6.2.

\subsection{Finite-source Effects and Other Constraints on $\theta_{\mathrm{E}}$}

\subsubsection{Color-Magnitude Diagram}

We follow the standard procedure to derive dereddened source color and magnitude from the color-magnitude diagram (CMD) of the observed field. Figure 3 shows the calibrated OGLE CMD (black), with the baseline source being displayed as a green point. The $V-I$ color of the source can be determined in 


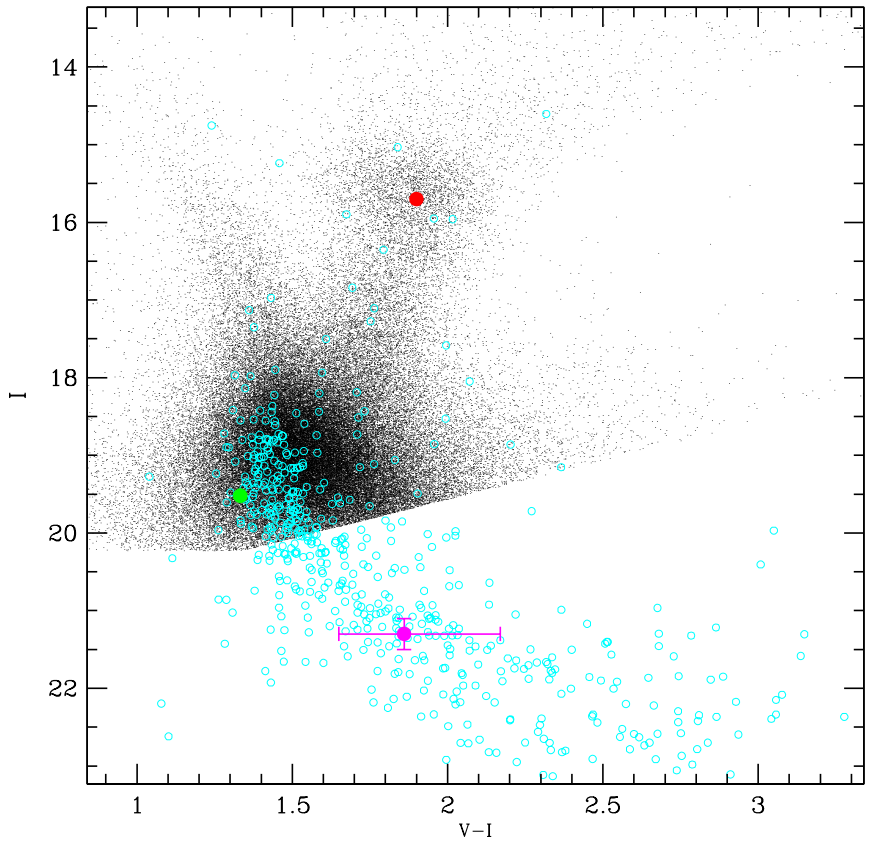

Figure 3. CMD for the OGLE-2005-BLG-071 field. Black dots are the stars with the OGLE $I$-band and $V$-band observations. The red point and green points show the center of red clump and the source, respectively. The errors in their fluxes and colors are too small to be visible on the graph. Cyan points are the stars in the ACS field, which are photometrically aligned with OGLE stars using 10 common stars. The magenta point with error bars show the color and magnitude of the blended light.

a model-independent way from linear regression of the $I$-band and $V$-band observations. The $I$-band magnitude of the source is also precisely determined from the microlens model, and it is hardly affected by any higher order effects. The center of red clump (red) is at $(V-I, I)_{\text {clump }}=(1.89,15.67)$. The Galactic coordinates of the source are at $(l, b)=(355.58,-3.79)$. Because the Galactic bulge is a barlike structure that is inclined relative to the plane of the sky, the red clump density at this sky position peaks behind the Galactic center by 0.15 mag (Nishiyama et al. 2005). Hence, we derive $(V-I, I)_{0, \text { clump }}=$ $(1.00,14.47)$, by adopting a Galactic distance $R_{0}=8 \mathrm{kpc}$. We thereby obtain the selective and total extinction toward the source $\left[E(V-I), A_{I}\right]=(0.89,1.20)$ and thus $R_{V I}=$ $A_{V} / E(V-I)=2.35$. The dereddened color and magnitude of the source is $((V-I), I)_{s, 0}=(0.45,18.31)$. From its dereddened color $(V-I)_{0}=0.45$, as well as its absolute magnitude (assuming it is in the bulge) $M_{I} \sim 3.65$, we conclude that the source is a main-sequence turnoff star. Following the method of Yoo et al. (2004), we transform $(V-I)_{0}=0.45$ to $(V-K)_{0}=0.93$ (Bessell \& Brett 1988), and based on the empirical relation between the color and surface brightness for subgiant and main-sequence stars (Kervella et al. 2004), we obtain the angular size of the source

$$
\theta_{*}=0.52 \times 10^{0.2\left(19.51-I_{s}\right)} \pm 0.05 \mu \mathrm{as},
$$

where $I_{s}$ is the apparent magnitude of the source in the $I$ band. Other features on the CMD shown in Figure 3 are further discussed in Section 3.5.

\subsubsection{Photometric Systematics of the Auckland Data Set}

The Auckland data set's excellent coverage over the two peaks makes it particularly useful for probing the finite-source effects. Unlike the more drastic "caustics crossings" that occur in some events, the finite-source effects during "cusp approaches" are relatively subtle. Hence, one must ensure that the photometry is not affected by systematics at the few percent level when determining $\rho=\theta_{*} / \theta_{\mathrm{E}}$. The Auckland photometry potentially suffers from two major systematic effects.

First, the photometry of constant stars reduced by $\mu$ FUN's DoPHOT pipeline are found to show sudden "jumps" of up to $\sim 10 \%$ when the field crossed the meridian each night. The signs and amplitudes of the "jumps" depend on the stars' positions on the CCD. The Auckland telescope was on a German equatorial mount, and hence the camera underwent a meridian flip. Due to scattered light, the flat-fielded images were not uniform in illumination for point sources, an effect that can be corrected by making "superflats" with photometry of constant stars (Manfroid 1995). We have constructed such "superflats" for each night of Auckland observations using 71 bright isolated comparison stars across the frame. The DoPHOT instrumental magnitude $m_{i, j}$ for star $i$ on frame $j$ is modeled by the following equation:

$$
m_{i, j}=m_{0, i}-f\left(x_{i, j}, y_{i, j}\right)-Z_{j}-f w h m_{i, j} \times s_{i},
$$

where $m_{0, i}$ is the corrected magnitude for star $i, f(x, y)$ is a biquartic illumination correction as a function of the $(x, y)$ position on the CCD frame with 14 parameters, $Z_{j}$ is a zeropoint parameter associated with each frame (but with $Z_{1}$ set to be zero), and $s_{i}$ is a linear correlation coefficient for the seeing $f w h m_{i, j}$. A least-squares fit that recursively rejects $4 \sigma$ outliers is performed to minimize $\chi^{2}$. The best-fit $f(x, y)$ is dominated by the linear terms and has small quadratic terms, while its cubic and quartic terms are negligible. The resulting reduced $\chi^{2}$ is close to unity, and the "jumps" for all stars are effectively eliminated. We apply the biquartic corrections to the images and then reduce the corrected images using the DIA pipeline. The resulting DIA photometry of the microlens target is essentially identical (at the $\sim 1 \%$ level) to that from the DIA reductions of the original Auckland images.

As we now show, this is because DIA photometry automatically removes any artifacts produced by the first- and secondorder illumination distortions if the sources are basically uniformly distributed across the frame. For the first-order effect, a meridian flip about the target (which is very close to center of the frame) will induce a change in the flux from the source, but it will also induce a change in the mean flux from all other stars in the frame, which for a linear correction will be the same as the change in the position of the "center of light" of the frame light. If the frame sources are uniformly distributed over the frame, the "center of light" will be the center of the frame, which is the same position as the source, therefore introducing no effects. The second-order transformation is even under a rotation of $180^{\circ}$, whereas a meridian flip is odd under this transformation. Hence, the flip has no effects at second order.

Second, the Auckland observations were unfiltered. The amount of atmospheric extinction differs for stars with different colors. As shown in Figure 3, the source is much bluer than most of the bright stars in the field, which dominate the reference image. So the amount of extinction for the source is different from the average extinction over the whole frame. This difference varies as the airmass changes over time during the observations. Coincidentally, the times of the two peaks were both near maximum airmass when the "differential extinction" effect is expected to be the most severe. To investigate this effect, we match the isolated stars in the Auckland frame with Cerro Tololo Inter-American Observatory (CTIO) $I$ and 
$V$ photometry. We identify 33 bright, reasonably isolated stars with $\left|(V-I)-(V-I)_{s}\right|<0.25$. We obtain a "light curve" for each of these stars, using exactly the same DIA procedure as for the source. We measure the mean magnitude of each of the 33 light curves and subtract this value from each of the 508 points on each light curve, thereby obtaining residuals that are presumably primarily due to air-mass variation. For each of the 508 epochs, we then take the mean of all of these residuals. We recursively remove outliers until all the remaining points are within $3 \sigma$ of the mean, as defined by the scatter of the remaining points. Typically, one or two of the 33 points are removed as outliers. The deviations are well fitted by a straight line,

$$
\frac{d \mathrm{Mag}}{d Z}=0.0347 \pm 0.0016
$$

where $Z$ is the air mass. The sense of the effect is that stars with the color of the microlensed source are systematically fainter at high air mass, as expected. (We also tried fitting the data to a parabola rather than a line, but the additional (quadratic) parameter was detected at substantially below $1 \sigma$.) Finally, we apply these "differential extinction" corrections to the "superflat"-adjusted DIA photometry to remove both photometric systematics.

In general, the finite-source effects depend on the limbdarkening profile of the source star in the observed passbands. We find below that in this case, the impact proves to be extremely weak. Nevertheless, using the matched Auckland and CTIO stars, we study the difference between Auckland magnitudes and $I$-band magnitude as a function of the $V-I$ color. We find the Auckland clear filter is close to the $R$ band.

\subsubsection{Blending in Palomar and MDM Data}

Palomar data cover only about 80 minutes, but these include the cresting of the second peak, from which we essentially derive all the information about finite-source effects. The Palomar data are sensitive to these effects through their curvature. The curvature derived from the raw data can be arbitrarily augmented in the fit (and therefore the finite-source effects arbitrarily suppressed) by increasing the blending. In general, the blending at any observatory is constrained by observations at substantially different magnifications, typically on different nights. However, no such constraints are available for Palomar, since observations were carried out on only one night.

We therefore set the Palomar blending $f_{b}=0.2 f_{s}$, that is, similar to the OGLE blending. That is, we assume that the observed flux variation of $9 \%$, over the Palomar night, actually reflects a magnification variation of $9 \% /\left[1-f_{b} / A f_{s}\right]=$ $9 \%+0.026 \%$, where $A \sim 70$ is the approximate magnification on that night. If our estimate of the blending were in error by of order unity (i.e., either $f_{b}=0$ or $f_{b}=0.4 f_{s}$ ), then the implied error in the magnification difference would be $0.026 \%$, which is more than an order of magnitude below the measurement errors. Hence, the assumption of fixed blending does not introduce "spurious information" into the fit even at the $1 \sigma$ level. MDM data cover the second peak for only $\sim 18$ minutes. For consistency, we treat its blending in the same way as Palomar, although the practical impact of this data set is an order of magnitude smaller.

\subsubsection{Modeling the Finite-source Effects}

After careful tests that are described immediately below, we determined that all finite-source calculations can be carried out to an accuracy of $10^{-4}$ using the hexadecapole approximation of Gould (2008; see also Pejcha \& Heyrovsky 2009). This sped up calculations by several orders of magnitude. We began by conducting MCMC simulations using the "loop linking" finitesource code described in Appendix A of Dong et al. (2006). From these simulations, we found the $4.5 \sigma$ upper bound on the finite-source parameter $\rho(4.5 \sigma)=0.001$. We then examined the differences between loop-linking (set at ultrahigh precision) and hexadecapole for light curves at this extreme limit and found a maximum difference of $10^{-4}$. Based on Claret (2000), we adopt linear limb-darkening coefficients $\Gamma_{I}=0.35$ for the $I$-band observations and $\Gamma_{R}=0.43$ for the observations performed in the $R$ band and the clear filters, where the local surface brightness is given by $S(\theta) \propto 1-\Gamma\left[1-1.5\left(1-\theta^{2} / \theta_{*}^{2}\right)^{1 / 2}\right]$. Ten additional MCMC runs are performed with $\Gamma_{I}$ and $\Gamma_{R}$ that differ from the above values by 0.1 or 0.2 . They result in essentially the same probability distributions of $\rho$. Therefore, the choice of limb-darkening parameters has no effect on the results. The source size is found to be $\rho=3.9_{-2.7}^{+1.8}$ for the wide solution and $\rho=3.1_{-2.5}^{+1.7}$ for the close solution. Solutions with $\rho>0.0009$ are ruled out at more than $3 \sigma$. The angular Einstein radius is given by $\theta_{\mathrm{E}}=\theta_{*} / \rho$. Hence, the lack of pronounced finite-source effects yields a $3 \sigma$ lower limit: $\theta_{\mathrm{E}}>0.6$ mas. The lens-source relative proper motion in the geocentric frame is simply $\mu_{\text {geo }}=\theta_{\mathrm{E}} / t_{\mathrm{E}}$. The posterior probability distributions of $\boldsymbol{\mu}_{\text {geo }}$ derived from these MCMC simulations are compared with those derived from astrometry in Section 3.4.

\subsection{HST Astrometry}

HST observations were taken at two epochs (HJD = 2453513.6 and HJD = 2453788.2) with the ACS High Resolution Camera (HRC). For each epoch, four dithered images were acquired in each of F814W and F555W with individual exposure times of $225 \mathrm{~s}$ and $315 \mathrm{~s}$ respectively. The position of the microlens on the HST frame is in excellent agreement with its centroid on the OGLE difference image (within $\sim 00^{\prime} 01$ ). The closest star to the source is about 0.'6 away. This implies that the OGLE photometry of the target star does not contain additional blended light that would be identifiable from the HST images. Data analysis was carried out using the software program img2xym_HRC (Anderson \& King 2004) in a manner similar to that described in Bennett et al. (2006). Stars are fitted with an empirical "library" PSF that was derived from well populated globular cluster fields. These positions are then corrected with precise distortion-correction models (accurate to $\sim 0.01$ pixel). We adopted the first F555W frame of the first epoch as the reference frame, and used the measured positions of stars in this frame and the frame of each exposure to define a linear transformation between the exposure frame and the reference frame. This allowed us to transform the position of the target star in each exposure into the reference frame, so that we see how the target star had moved relative to the other stars. The centroid positions of the target star in each filter and epoch are shown in Figure 4. For convenience, in this figure, the positions are displayed relative to the average of the centroid positions. The error bars are derived from the internal scatter of the four dithered images. The probability is $P=38 \%$ of measuring the observed separation (or larger) between F814W and F555W under the assumption that the true offset is zero. The fact that the blended light is aligned with the source argues that it is associated with the event (either it is the lens itself or a companion to the lens or the source). We give a more quantitative statement of this 


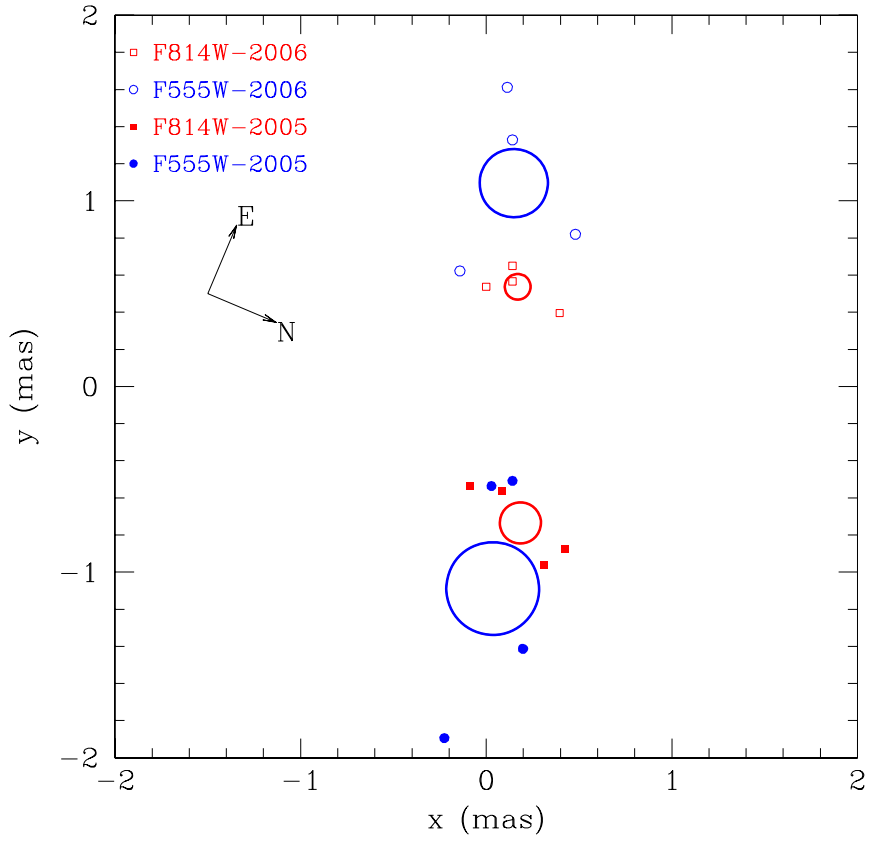

Figure 4. HST ACS astrometric measurements of the target star in F814W (red) and F555W (blue) filters in 2005 (filled dots) and 2006 (open dots). The center positions of the big circles show mean values of the four dithered observations in each filter at each epoch while radii of the circle represent the $1 \sigma$ errors.

constraint in Section 3.5. For the present, we simply note that the $P=38 \%$ probability is compatible with the picture that the blend is due to the lens since the first epoch was only about half of the Einstein-radius crossing time after $t_{0}$, implying that the lens-source separation induces only a very small centroid offset, well below the HST detection limit. For the second epoch, the centroid offset is

$$
\begin{aligned}
\Delta \mathbf{r}_{\mathrm{F} 814 \mathrm{~W}-\mathrm{F} 555 \mathrm{~W}, \text { east }} & =-0.52 \pm 0.20 \text { mas, } \\
\Delta \mathbf{r}_{\mathrm{F} 814 \mathrm{~W}-\mathrm{F} 555 \mathrm{~W}, \text { north }} & =0.22 \pm 0.20 \text { mas. }
\end{aligned}
$$

We also calculate the error in the centroid offsets from the scatter in such offsets among all comparison stars with F555W magnitudes within $0.5 \mathrm{mag}$ of the target and find that it is consistent with the internally based error quoted above.

At the peak of the event, the angular separation between the lens and the source was negligible, since $u_{0} \ll 1$. We therefore fix the angular positions of the lens and source at a common $\boldsymbol{\theta}_{\mathbf{0}}$. From the CMD (Figure 3), most of the stars in the HST field are from the bulge. So we set a reference frame that is fixed with respect to the bulge field at distance $D_{s}$. The source and lens positions at time $t$ are then

$$
\begin{aligned}
& \boldsymbol{\theta}_{\boldsymbol{s}}(t)=\boldsymbol{\theta}_{0}+\mu_{\boldsymbol{s}}\left(t-t_{0}\right), \\
& \boldsymbol{\theta}_{\boldsymbol{l}}(t)=\boldsymbol{\theta}_{\mathbf{0}}+\boldsymbol{\mu}_{\boldsymbol{l}}\left(t-t_{0}\right)+\pi_{\mathrm{rel}}\left[s(t)-\boldsymbol{s}\left(t_{0}\right)\right],
\end{aligned}
$$

where $s(t)$ is the Earth-to-Sun vector defined by Gould (2004). Then by applying Equations (3) and (4), the angular separation between the lens and source is

$$
\boldsymbol{\theta}_{\text {rel }}(t)=\boldsymbol{\theta}_{\boldsymbol{l}}(t)-\boldsymbol{\theta}_{\boldsymbol{s}}(t)=\boldsymbol{\mu}_{\mathrm{geo}}\left(t-t_{0}\right)+\pi_{\mathrm{rel}} \Delta \boldsymbol{s}(t),
$$

where $\Delta s(t)$ is given by Equation (5) in Gould (2004).

The centroid of the source images $\boldsymbol{\theta}_{s}^{\prime}$ is displaced from the source position by (Walker 1995),

$$
\Delta \boldsymbol{\theta}_{s}(t)=\boldsymbol{\theta}_{\boldsymbol{s}}^{\prime}(t)-\boldsymbol{\theta}_{\boldsymbol{s}}(t)=\frac{-\boldsymbol{\theta}_{\mathrm{rel}}(t)}{\left[\theta_{\mathrm{rel}}(t) / \theta_{\mathrm{E}}\right]^{2}+2} .
$$

Therefore, one can obtain the centroid position of the lens and the source at time $t$ :

$$
\begin{aligned}
\boldsymbol{\theta}_{\boldsymbol{c}}(t) & =\left[1-f_{l}(t)\right]\left[\boldsymbol{\theta}_{\boldsymbol{s}}(t)+\boldsymbol{\Delta} \boldsymbol{\theta}_{s}(t)\right]+f_{l}(t) \boldsymbol{\theta}_{\boldsymbol{l}}(t) \\
& =\boldsymbol{\theta}_{\mathbf{0}}+\boldsymbol{\mu}_{\boldsymbol{s}}\left(t-t_{0}\right)+\boldsymbol{\theta}_{\mathrm{rel}}(t)\left[f_{l}(t)+\frac{1-f_{l}(t)}{\left[\theta_{\mathrm{rel}}(t) / \theta_{\mathrm{E}}\right]^{2}+2}\right],
\end{aligned}
$$

where $f_{l}(t)$ is the fraction of the total flux due to the lens.

The centroid offset between the two passbands, F814W and $\mathrm{F} 555 \mathrm{~W}$, is related to the properties of the system by

$$
\begin{aligned}
\Delta \boldsymbol{\theta}_{c}(t)_{\mathrm{F} 814 \mathrm{~W}-\mathrm{F} 555 \mathrm{~W}} & =\left[f(t)_{l, \mathrm{~F} 814 \mathrm{~W}}-f(t)_{l, \mathrm{~F} 555 \mathrm{~W}}\right] \\
& \times\left[1-\frac{1}{\left[\theta_{\mathrm{rel}}(t) / \theta_{\mathrm{E}}\right]^{2}+2}\right] \boldsymbol{\theta}_{\mathrm{rel}}(t) .
\end{aligned}
$$

The difference of the blend's fractional flux between F814W and F555W is obtained from "MCMC A" described in Section 3.5. Consequently, under the assumption that the blend is the lens, we can use the measurement of the second-epoch HST centroid offset to estimate the relative proper motion from Equation (15) for a given $\pi_{\text {rel }}$. For purposes of illustration, we temporarily adopt $\pi_{\text {rel }}=0.2$ when calculating the probability distribution of $\boldsymbol{\mu}_{\text {geo }}$ (black contours in the upper panels of Figure 5). The centroid shift generally favors faster relative proper motion than that derived from the source size measurement (green contours in Figure 5), but the difference is only at the $\sim 1 \sigma$ level. We then get a joint probability distribution of $\boldsymbol{\mu}_{\text {geo }}$ from both finite-source effects and astrometry, which is shown as the red contours in the upper panels of Figure 5.

We then derive the distribution of the $\boldsymbol{\mu}_{\text {geo }}$ position angle (P.A.) $\phi \boldsymbol{\mu}_{\text {geo }}$ (north through east), which is shown by the red histograms in the lower panels of Figure 5. Since the direction of the lens-source relative proper motion $\boldsymbol{\mu}_{\mathrm{geo}}$ is the same as that of the microlens parallax $\pi_{\mathrm{E}}$ in the geocentric frame, we have an independent check on the $\phi_{\mu_{\text {geo }}}$ from our parallax measurements, whose distribution is plotted as blue histograms in Figure 5. Both constraints favor the lens-source proper motion to be generally west, but they disagree in the northsouth component for which both constraints are weaker. The disagreements between two histograms is at about $2.5 \sigma$ level.

\section{5. "Seeing" the Blend with HST}

If the blend were not the lens (or otherwise associated with the event), the PSF of the source would likely be broadened by the blended star. We examine the HST F814W images of the target and 45 nearby stars with similar brightness for each available exposure. We fit them with the library PSF produced by Anderson \& King (2004). In order to account for breathingrelated changes of focus, we fit each of these 45 nearby stars with the library PSF, and construct a residual PSF that can be added to the library PSF to produce a PSF that is tailor-made for each exposure. For both epochs, the source-blend combination shows no detectable broadening relative to the PSFs of other isolated stars in the field. From the ground-based light curve, it is already known that $\sim 16 \%$ of this light comes from the blend. We add simulated stars with the same flux as the blended light from 0 to 2.0 pixels away from the center of the source. We find that the blend would have produced detectable broadening of the PSF if it were more than 15 mas apart from the source at the second epoch. Hence, the source-blend separation must then be less than about 15 mas. From the HST image itself, the density of ambient stars at similar magnitudes is $\lesssim 1 \operatorname{arcsec}^{-2}$. The 

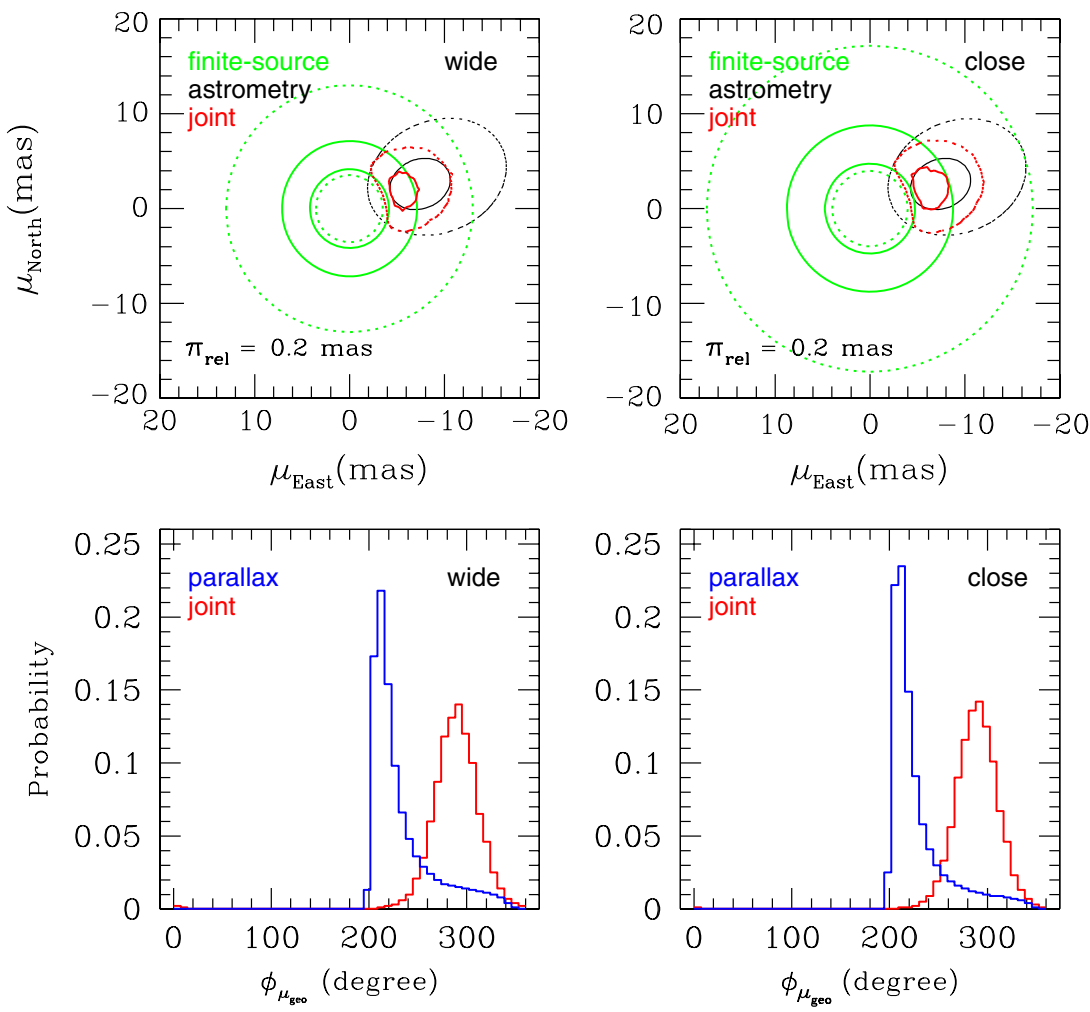

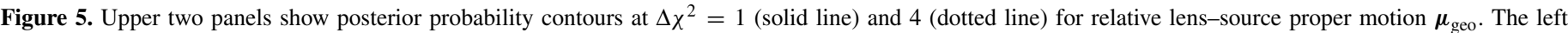

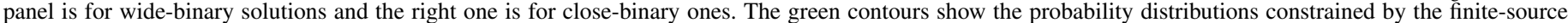

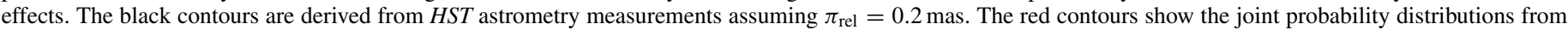

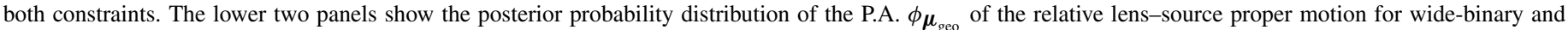

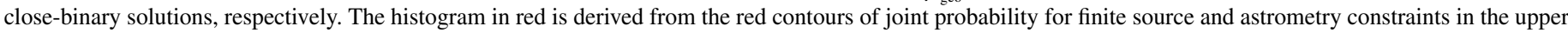
panel. The blue histogram represents that of the microlens parallax. They mildly disagree at $2.5 \sigma$.

probability of a chance interloper is therefore less than $0.07 \%$, implying that the blended light is almost certainly associated with the event, that is, either the lens itself, a companion to the lens, or a companion to the source. Both of the latter options are further constrained in Section 3.7 where, in particular, we essentially rule out the lens-companion scenario.

As discussed in Section 2, the blended flux in $I$ is relatively well determined from the ground-based OGLE data alone, but the blended $V$ flux is poorly determined, primarily because the systematic uncertainty in the zero point of the baseline flux (determined from PSF fitting) is of the same order as the blended flux. Because the HST image is very sparse, there is essentially no zero-point error in the HST $V$-band flux. The problem is how to divide the baseline $V$ flux into source and blend fluxes, $F_{\text {base }}=F_{s}+F_{b}$.

The standard method of doing this decomposition would be to incorporate the HST $V$ light curve into the overall fit, which would automatically yield the required decomposition. Since this "light curve" consists of two points, the "fit" can be expressed analytically

$$
F_{s}=\frac{F\left(t_{1}\right)-F\left(t_{2}\right)}{A_{1}-1}, \quad F_{b}=F\left(t_{2}\right)-F_{s},
$$

where we have made the approximation that the second observation is at baseline. Let us then estimate the resulting errors in $F_{s}$ and $F_{b}$, ignoring for the moment that there is some uncertainty in $A_{1}$ due to uncertainties in the general model. Each of the individual flux measurement is determined from four separate subexposures, and this permits estimates of the errors from the respective scatters. These are $\sigma_{1}=0.01$ and $\sigma_{2}=0.03 \mathrm{mag}$. Hence, the fractional error in $F_{s}$ is $(2.5 / \ln 10) \sigma\left(F_{s}\right) / F_{s}=\left[\sigma_{1}^{2}\left(A_{1}+r\right)^{2}+\sigma_{2}^{2}(1+r)^{2}\right]^{1 / 2} /\left(A_{1}-1\right)$, where $r \equiv F_{b} / F_{s}$. Adopting, for purposes of illustration, $A_{1}=2$ and $r=0.1$, this implies an error $\sigma\left(V_{s, H S T}\right)$ of 0.04 mag. This may not seem very large, but after the subtraction in Equation (16), it implies an error $\sigma\left(V_{b, H S T}\right) \sim \sigma\left(V_{s, H S T}\right) / r \sim 0.4$ mag. And taking into account the uncertainties introduced by model fitting in determining the magnifications, the error is expected to be even larger. Hence, we undertake an alternate approach.

Because the HST and OGLE $V$ filters have very nearly the same wavelength center, $V_{S, H S T}$ should be nearly identical to $V_{s, \text { OGLE }}$ up to a possible zero-point offset on their respective magnitude scales. Because the OGLE data contain many more points during the event, some at much higher magnification than the single $H S T$ event point, $V_{s, \text { OGLE }}$ is determined extremely well (for a fixed microlensing model), much better than the 0.04 mag error for $V_{s, H S T}$. Thus, if the zero-point offset between the two systems can be determined to better than 0.04 mag, this method will be superior. Although the I-band blend is much better measured than the $V$-band blend from the ground-based data, for consistency we determine the zero-point offset in $I$ by the same procedure.

Figure 6 shows differences between OGLE and HST $V$ magnitudes for matched stars in the HST image. The error for each star and observatory is determined from the scatter among measurements of that star. We consider only points with $V<19.5$ because at fainter magnitudes the scatter grows 
considerably. Each star was inspected on the HST images, and those that would be significantly blended on the OGLE image were eliminated. The remaining points are fit to an average offset by adding a "cosmic error" in quadrature to the errors shown. We carry out this calculation twice, once including the "outlier" (shown as a filled circle) and once with this object excluded. For the $V$ band, we find offsets of $V_{H S T}-V_{\text {OGLE }}=0.17 \pm 0.01$ and $0.18 \pm 0.01$, respectively. We adopt the following $V$-band offset

$$
\Delta V=V_{H S T}-V_{\mathrm{OGLE}}=0.18 \pm 0.01
$$

A similar analysis of the $I$ band leads to

$$
\Delta I=I_{H S T}-I_{\mathrm{OGLE}}=0.08 \pm 0.01
$$

We find no obvious color terms for either the $V$-band or $I$-band transformations. As a check, we perform linear regression to compare the OGLE and HST $(V-I)$ colors, and we find they agree within $0.01 \mathrm{mag}$, which further confirms the color terms are unlikely to be significant in the above transformations.

We proceed as follows to make $H S T$-based MCMC ("MCMC A") estimates of $V_{b, \text { OGLE }}$ and $I_{b, \text { OGLE }}$ that place the blending star on the OGLE-based CMD. Since flux parameters are linear, they are often left free and fitted by linear leastsquares minimization, which significantly accelerates the computations. However, for "MCMC A," the source fluxes from OGLE and HST are treated as independent MCMC parameters so that they can help align the two photometric systems as described below. Since HST blended light is not affected by light from ambient stars (as OGLE is), we also leave HST blended fluxes as independent. Therefore, in "MCMC A," we include the following independent MCMC flux parameters, $F_{I, s, \text { OGLE, }}$ $F_{V, s, \text { OGLE }}, F_{I, s, H S T}, F_{V, s, H S T}, F_{I, b, H S T}$, and $F_{V, b, H S T}$, which for convenience we express here as magnitudes. For each model on the chain, we add to the light-curve-based $\chi^{2}$ two additional terms $\Delta \chi_{V}^{2}=\left(V_{s, H S T}-V_{s, \mathrm{OGLE}}-\Delta V\right)^{2} /[\sigma(\Delta V)]^{2}$ and $\Delta \chi_{I}^{2}=\left(I_{s, H S T}-I_{s, \mathrm{OGLE}}-\Delta I\right)^{2} /[\sigma(\Delta I)]^{2}$ to enforce the measured offset between the two systems. Finally, we evaluate the $V$-band blended flux from $H S T$ and convert it to OGLE system, $V_{b, \mathrm{OGLE} / H S T}=V_{s, \mathrm{OGLE}}-V_{s, H S T}+V_{b, H S T}$ (and similarly for the $I$ band), where all three terms on the right-hand side are the individual Monte Carlo realizations of the respective parameters.

The result is shown in Figure 3, in which the blend (magenta) is placed on the OGLE CMD. Also shown, in cyan points, is $H S T$ photometry (aligned to the OGLE system) of the stars in the ACS subfield of the OGLE field. Although this field is much smaller, its stars trace the main sequence to much fainter magnitudes. The blend falls well within the bulge main sequence revealed by the $H S T$ stars on the CMD, so naively the blend can be interpreted as being in the bulge. Hence, this diagram is, in itself, most simply explained by the blend being a bulge lens or a binary companion of the source. However, the measurement of $V-I$ color has relatively large uncertainty, and it is also consistent with the blend being the lens (or a companion to it) several kpc in front of the bulge, provided the blend is somewhat redder than indicated by the best-fit value of its color. In Section 3.6, we assume the blended light seen by HST is the foreground lens star, and the HST photometry is combined with other information to put constraints on the lens star under this assumption.

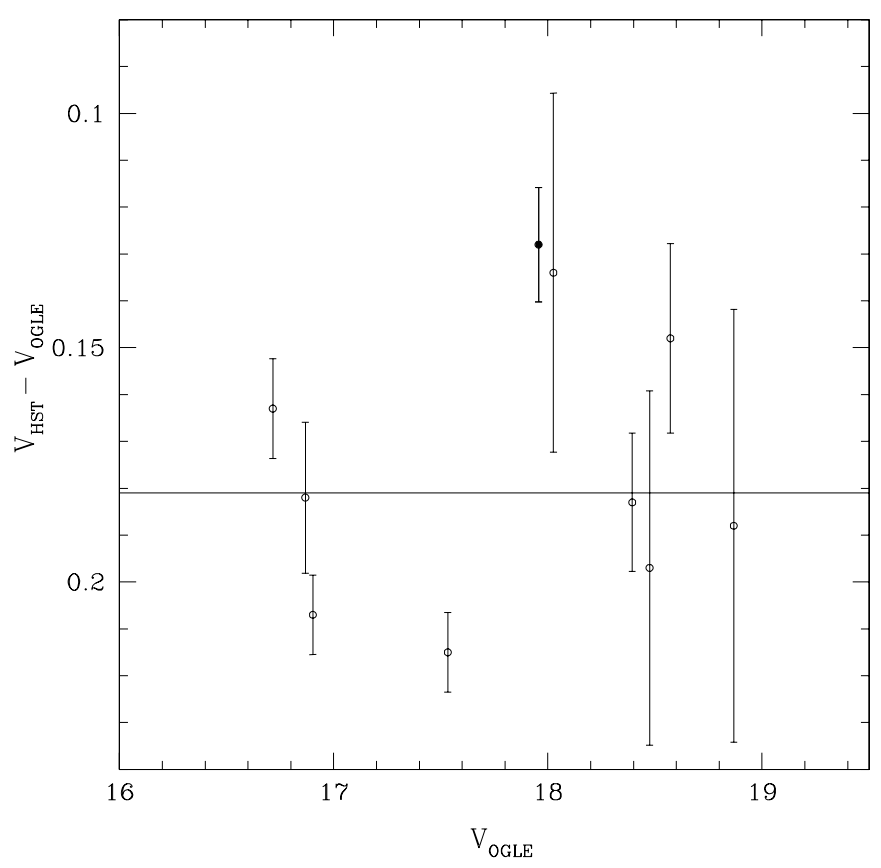

Figure 6. Differences between OGLE $V$ and HST F555W magnitudes for the matched stars are plotted against their $V$ magnitudes measured by OGLE. To calculate the offset, we add a 0.017 mag "cosmic error" in quadrature to each point in order to reduce $\chi^{2} /$ dof to unity. The open circles represent the stars used to establish the final transformation and the filled point shows an "outlier."

\subsection{Final Physical Constraints on the Lens and Planet}

\subsubsection{Constraints on a Luminous Lens}

In the foregoing, we have discussed two types of constraints on the host star properties: the first class of constraints, consisting of independent measurements of $\boldsymbol{\pi}_{\mathbf{E}}, \theta_{\mathrm{E}}$, and $\boldsymbol{\mu}$, relate the microlens parameters to the physical parameters of the lens; the second class are HST and ground-based observations that determine the photometric properties of the blend.

In this section, we first describe a new set of MCMC simulations taking all these constraints into account. Similarly to what is done to include HST photometry in the "MCMC A" (see Section 3.5), we incorporate HST astrometry constraints by adding $\chi^{2}$ penalties to the fittings. For a given set of microlens parameters, we can derive the physical parameters, namely, $M$, $\pi_{\text {rel }}, \boldsymbol{\mu}_{\text {geo }}$, and so calculate $\rho=\theta_{*} / \theta_{\mathrm{E}}$ (from Equation (1)) and the F814W - F555W centroid offset (from Equation (15)). Then we assign the $\chi^{2}$ penalties based on the observed centroid offset from Section 3.4. In this way, the MCMC simulations simultaneously include all microlens constraints on the lens properties. The posterior probability distribution of $M$ and $\pi_{\text {rel }}$ are plotted in Figure 7 . The $\pi_{\text {rel }}$ determination very strongly excludes a bulge $\left(\pi_{\text {rel }} \lesssim 0.05\right)$ lens. Note that by incorporating HST astrometry, we implicitly assume that the blend is the lens.

If the blend is indeed the lens itself, we can also estimate its mass and distance from the measured color and magnitude of the blend. In doing so, we use theoretical stellar isochrones (M. Pinsonneault 2007, private communication) incorporating the color-temperature relation by Lejeune et al. (1997, 1998). We first use an isochrone that has solar metal abundance, with stellar masses ranging from $0.25 M_{\odot}-1.0 M_{\odot}$, and an age of 4 Gyr. The variation in stellar brightness due to stellar age is negligible for our purpose. Extinction is modeled as a function of $D_{l}$ by $d A_{I} / d D_{l}=\left(0.4 \mathrm{kpc}^{-1}\right) \exp \left(-w D_{l}\right)$, where $w$ is set to be $0.31 \mathrm{kpc}^{-1}$ so that the observed value $A_{I}(8.6 \mathrm{kpc})=1.20($ as 


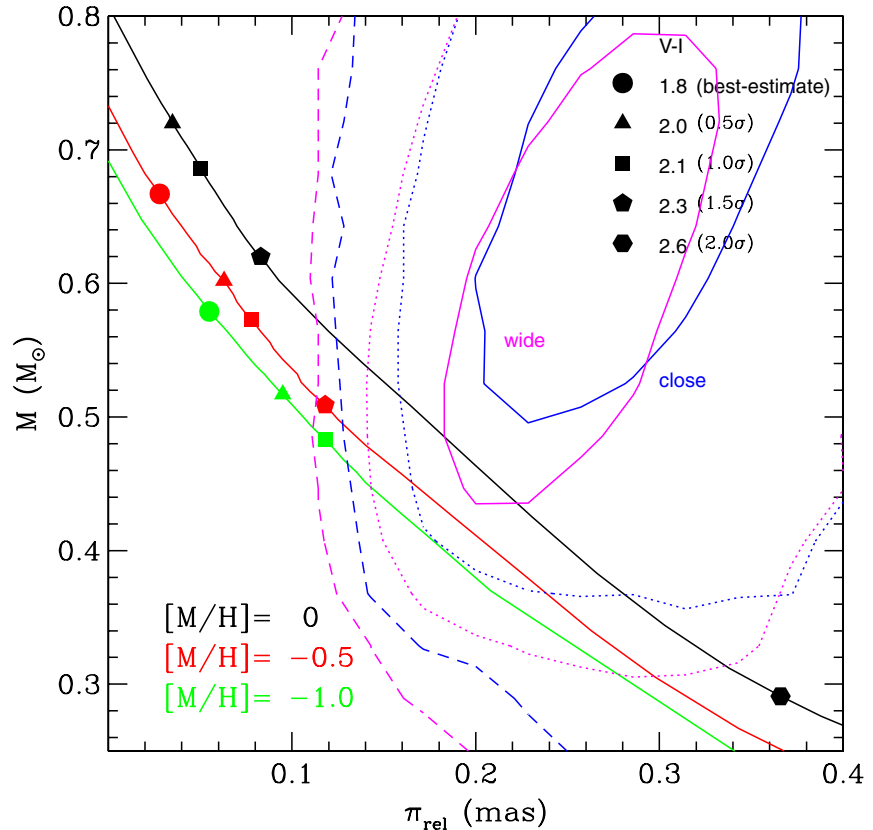

Figure 7. Posterior probability distribution of lens mass $M$, and relative lenssource parallax $\pi_{\text {rel }}$ from MCMC simulations discussed in Section 3.6.1. The constraints include those from parallax effects, finite-source effects and relative proper-motion measurements from $H S T$ astrometry. The $\Delta \chi^{2}=1,4,9$ contours are displayed in solid, dotted, and dashed lines, respectively. Both wide-binary (magenta) and close-binary (blue) solutions are shown. The lines in black, red, and green represent the predicted $M$ and $\pi_{\text {rel }}$ from the isochrones for different metal abundances: $[\mathrm{M} / \mathrm{H}]=0$ (black), -0.5 (red), -1.0 (green). The points on these lines correspond to the observed $I$-band magnitude $I=21.3$ and various $V-I$ values $V-I=1.8$ (best estimate, filled dots), 2.0 ( $0.5 \sigma$, filled triangle),

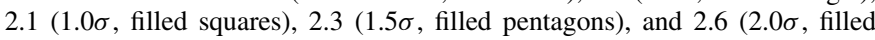
hexagons).

derived from CMD discussed in Section 3.3.1) is reproduced. Again, the distance to the source is assumed to be $8.6 \mathrm{kpc}$, implying $\pi_{s}=0.116$ mas, and hence that the lens distance is $D_{l} / \mathrm{kpc}=\operatorname{mas} /\left(\pi_{\mathrm{rel}}+\pi_{s}\right)$. In Figure 7 , we show the lens mass $M$ and relative parallax $\pi_{\text {rel }}$ derived from the isochrone that correspond to the observed $I$-band magnitude $I=21.3$ in black line and a series of $V-I$ values $V-I=1.8$ (best estimate), $2.0(0.5 \sigma), 2.1(1 \sigma), 2.3(1.5 \sigma)$ and $2.6(2 \sigma)$ as black points. The observed color is in modest disagreement $<2 \sigma$ with the mass and distance of the lens at solar metallicity. We also show analogous trajectories for $[\mathrm{M} / \mathrm{H}]=-0.5$ (red) and $[\mathrm{M} / \mathrm{H}]=-1.0$ (green). The level of agreement changes only very weakly with metallicity.

We then include the isochrone information in a new set of MCMC runs ("MCMC B"). To do so, the HST blended fluxes in $I$ and $V$ bands can no longer be treated as independent MCMC parameters. Instead, based on the isochrone with solar metallicity, the lens $V-I$ color and $I$ magnitude are predicted at the lens mass and distance determined from MCMC parameters. Then the HST I-band and $V$-band fluxes are fixed at the predicted values in the fitting for each MCMC realization.

Figure 8 illustrates the constraints on $M$ and $\pi_{\text {rel }}$ from the MCMC, which are essentially the same for both wide-binary (solid contours) and close-binary (dashed contours) solutions:

$$
M=0.46 \pm 0.04 M_{\odot}, \quad \pi_{\mathrm{rel}}=0.19 \pm 0.03 \text { mas. }
$$

Assuming the source distance at $8.6 \mathrm{kpc}$, the $\pi_{\text {rel }}$ estimates translate to the following lens distance measurement:

$$
D_{l}=3.2 \pm 0.4 \mathrm{kpc}
$$

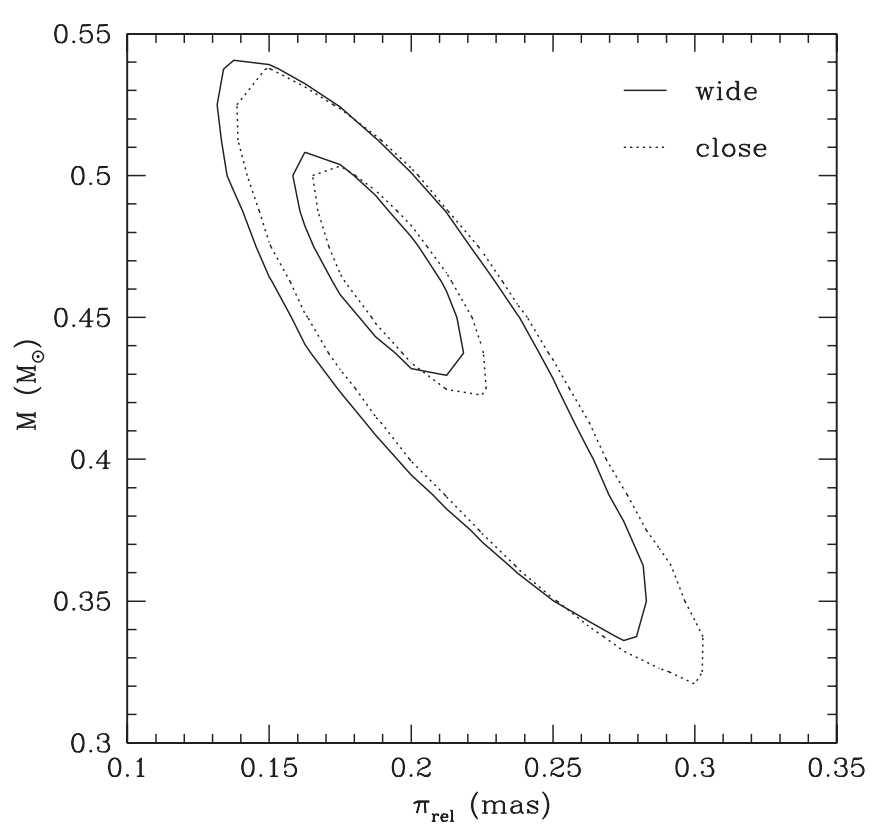

Figure 8. Posterior probability distribution of lens mass $M$ and relative lenssource parallax $\pi_{\text {rel }}$ from MCMC simulations assuming that the blended light comes from the lens star. The $\Delta \chi^{2}=1,4$ contours are displayed in a solid line for wide solutions and in a dotted line for close solutions.

Furthermore, we can derive constraints on the planet mass $M_{p}$ and the projected separation between the planet and the lens star $r_{\perp}$

$M_{p}=3.8 \pm 0.4 M_{\mathrm{Jupiter}}, \quad r_{\perp}=3.6 \pm 0.2 \mathrm{AU} \quad$ (wide),

and

$M_{p}=3.4 \pm 0.4 M_{\text {Jupiter }}, \quad r_{\perp}=2.1 \pm 0.1 \mathrm{AU} \quad$ (close).

The wide solution is slightly preferred over close solution by $\Delta \chi^{2}=2.1$.

To examine possible uncertainties in extinction estimates, we reran our MCMC with $A_{I}$ and $A_{V}$ that are $10 \%$ higher and lower than the fiducial values. These runs result in very similar estimates as when adopting the fiducial values.

From Equations (14) and (13), one can easily obtain the centroid shift between two epochs in a given passband by ignoring $\boldsymbol{\Delta} \boldsymbol{\theta}_{s}(t),{ }^{47}$

$$
\begin{aligned}
\boldsymbol{\theta}_{\boldsymbol{c}}\left(t_{2}\right)-\boldsymbol{\theta}_{\boldsymbol{c}}\left(t_{1}\right)= & \boldsymbol{\mu}_{\boldsymbol{s}}\left(t_{2}-t_{1}\right)+\boldsymbol{\mu}_{\mathrm{geo}} \\
& \times\left[f_{l}\left(t_{2}\right)\left(t_{2}-t_{0}\right)-f_{l}\left(t_{1}\right)\left(t_{1}-t_{0}\right)\right] \\
& +\pi_{\mathrm{rel}}\left[f_{l}\left(t_{2}\right) \Delta \boldsymbol{s}\left(t_{2}\right)-f_{l}\left(t_{1}\right) \Delta \boldsymbol{s}\left(t_{1}\right)\right] .
\end{aligned}
$$

Because $\boldsymbol{\mu}_{\mathrm{geo}}, \pi_{\text {rel }}$ and $f_{l}$ in a given passband can be extracted from the MCMC realizations ("MCMC B"), we can use the above equation to measure the source proper motion by making use of the centroid shift in F814W between two epochs. The source proper motion with respect to the mean motion of stars

\footnotetext{
47 The angular separations between the source and the lens are $\sim 0.47 \theta_{\mathrm{E}}$ and $\sim 4.4 \theta_{\mathrm{E}}$ for the two $H S T$ epochs, respectively. Thus, the angular position offsets between the centroids of the source images and the source are both $\sim 0.21 \theta_{\mathrm{E}}$ and the directions of the offset relative to the source are almost the same due to the small impact parameter $u_{0}$. The difference between lens flux fractions of the two epochs are about $7 \%$ in the $I$ band, so the offsets can be confidently ignored in deriving the source proper motion using the relative astrometry in F814W at two different epochs.
} 
in the HST field is measured to be

$$
\boldsymbol{\mu}_{s}=\left(\mu_{s, E}, \mu_{s, N}\right)=\left(2.0 \pm 0.2,-0.5_{-0.7}^{+0.2}\right) \operatorname{mas~yr}^{-1} .
$$

We obtain similar results with F555W, but with understandably larger errorbars since the astrometry is more precise for the microlens in F814W.

Combining Equations (3) and (4), the lens proper motion in the heliocentric frame is therefore

$$
\boldsymbol{\mu}_{l}=\boldsymbol{\mu}_{\mathrm{geo}}+\boldsymbol{\mu}_{s}+\frac{\boldsymbol{v}_{\oplus} \pi_{\mathrm{rel}}}{\mathrm{AU}} .
$$

For each MCMC realization, $\pi_{\text {rel }}$ is known, so we can convert the lens proper motion to the velocity of the lens in the heliocentric frame $\boldsymbol{v}_{l, \text { hel }}$ and also in the frame of local standard of rest $\boldsymbol{v}_{l, \mathrm{LSR}}$ (we ignore the rotation of the galactic bulge). The lens velocity in the LSR is estimated to be $v_{l, \mathrm{LSR}}=103 \pm 15 \mathrm{~km} \mathrm{~s}^{-1}$. This raises the possibility of the lens being in the thick disk, in which the stars are typically metal-poor. As shown in Figure 7, the constraints we have cannot resolve the metallicity of the lens star.

\subsubsection{Planetary Orbital Motion}

Wide/Close Degeneracy. Binary-lens light curves in general exhibit a well-known "close-wide" symmetry (Dominik 1999; An 2005). Even for some well covered caustics-crossing events (e.g., Albrow et al. 1999), there are quite degenerate sets of solutions between wide and close binaries. In Paper I, we found that the best-fit point-source wide-binary solution was preferred over close-binary solutions by $\Delta \chi^{2} \sim 22$. But this did not necessarily mean that the wide-close binary degeneracy was broken, since the two classes of binaries may be influenced differently by higher order effects. We find that the $\chi^{2}$ difference between best-fit wide and close solutions is within 1 from "MCMC A" and 2.1 (positive $u_{0}$ ) or 2.2 (negative $u_{0}$ ) from "MCMC B."

However, orbital motion of the planet is subject to additional dynamical constraints: the projected velocity of the planet should be no greater than the escape velocity of the system: $v_{\perp} \leqslant v_{\text {esc }}$, where,

$$
\begin{gathered}
v_{\perp}=\sqrt{\dot{d}^{2}+(\omega d)^{2}} \frac{\mathrm{AU}}{\pi_{l}} \theta_{\mathrm{E}} \\
v_{\mathrm{esc}}=\sqrt{\frac{2 G M}{r}} \leqslant v_{\mathrm{esc}, \perp} \equiv \sqrt{\frac{2 G M}{d \theta_{\mathrm{E}} D_{l}}}=\sqrt{\frac{\pi_{l}}{2 d \pi_{\mathrm{E}}}} c,
\end{gathered}
$$

and where $r$ is the instantaneous three-dimensional planet-star physical separation. Note that in the last step, we have used Equation (1).

We then calculate the probability distribution of the ratio

$$
\frac{v_{\perp}^{2}}{v_{\mathrm{esc}, \perp}^{2}}=2 \frac{\mathrm{AU}^{2}}{c^{2}} \frac{d^{3}\left[(\dot{d} / d)^{2}+\omega^{2}\right]}{\left[\pi_{\mathrm{E}}+\left(\pi_{s} / \theta_{\mathrm{E}}\right)\right]^{3}} \frac{\pi_{\mathrm{E}}}{\theta_{\mathrm{E}}}
$$

for an ensemble of MCMC realizations for both wide and close solutions. Figure 9 shows probability distributions of the projected velocity $r_{\perp} \boldsymbol{\gamma}$ in the units of critical velocity $v_{\mathrm{c}, \perp}$, where $r_{\perp} \boldsymbol{\gamma}$ is the instantaneous velocity of the planet on the sky, which is further discussed in the Appendix and $v_{\mathrm{c}, \perp}=v_{\mathrm{esc}, \perp} / \sqrt{2}$. The dotted circle encloses the solutions that are allowed by the escape velocity criteria, and the solutions that are inside the solid line are consistent with circular orbital motion. We find
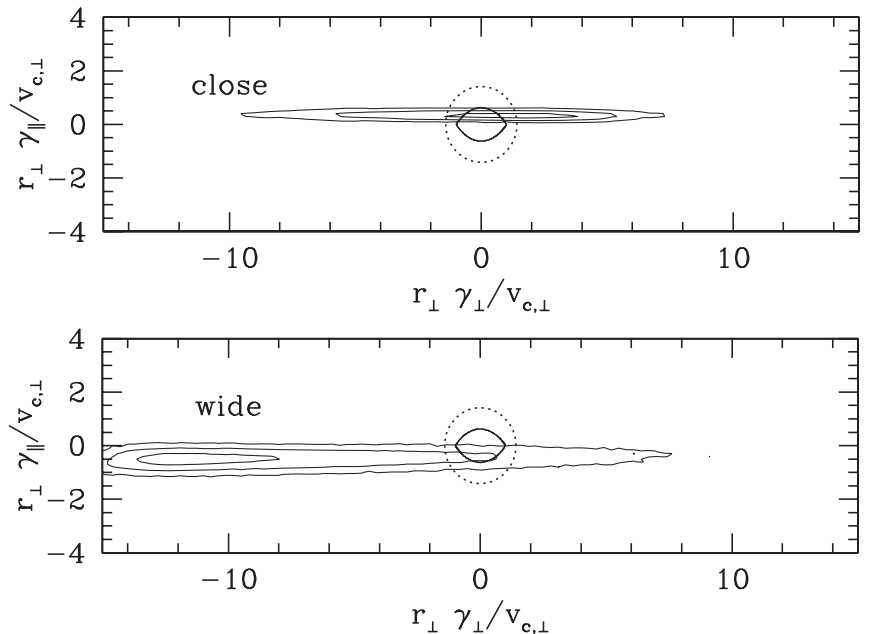

Figure 9. Probability contours of projected velocity $r_{\perp} \gamma$ (defined in the Appendix) in the units of $v_{\mathrm{c}, \perp}$ for both close-binary (upper panel) and widebinary (lower panel) solutions. All the solutions that are outside the dotted circle are physically rejected as the velocities exceed the escape velocity of the system. The boundary in a solid line inside the dotted circle encloses the solutions for which circular orbits are allowed.

that the best-fit close-binary solutions are physically allowed while the best-fit wide-binary solutions are excluded by these physical constraints at $1.6 \sigma$. The physically excluded best-fit wide solutions are favored by $\Delta \chi^{2}=2.1$ (or 2.2) over the close solutions, so by putting physical constraints, the degenerate solutions are statistically not distinguishable at $1 \sigma$.

Circular Planetary Orbits and Planetary Parameters. Planetary deviations in microlensing light curves are intrinsically short, so in most cases, only the instantaneous projected distance between the planet and the host star can be extracted. As shown in Section 3.6.2, for this event, we tentatively measure the instantaneous projected velocity of the planet, thanks to the relatively long ( $\sim 4$ days) duration of the planetary signal. One cannot solve for the full set of orbital parameters just from the instantaneous projected position and velocity. However, as we show in the Appendix, we can tentatively derive orbital parameters by assuming that the planet follows a circular orbit around the host star. In Figure 10, we show the probability distributions of the semimajor axis, inclination, amplitude of radial velocity (RV), and equilibrium temperature of the planet derived from "MCMC B" for both wide and close solutions. The equilibrium temperature is defined to be $T_{\text {eq }} \equiv\left(L_{\text {bol }} / L_{\text {bol, } \odot}\right)^{1 / 4}\left(2 a / R_{\odot}\right)^{-1 / 2} T_{\odot}$, where $L_{\text {bol }}$ is the bolometric luminosity of the host, $a$ is the planet semimajor axis, and $L_{\mathrm{bol}, \odot}, R_{\odot}$, and $T_{\odot}$ are the luminosity, radius, and effective temperature of the Sun, respectively. This would give the Earth an equilibrium temperature of $T_{\mathrm{eq}}=285 \mathrm{~K}$. In calculating these probabilities, we assign a flat (Öpik's Law) prior for the semimajor axis and assume that the orbits are randomly oriented, that is, with a uniform prior on $\cos i$.

\subsection{Constraints on a Nonluminous Lens}

In Section 3.5, we noted that the blended light must lie within 15 mas of the source: otherwise, the HST images would appear extended. We argued that the blended light must be associated with the event (either the lens itself or a companion to either the source or lens), since the chance of such an alignment by a random field star is less than $0.07 \%$. In fact, even stronger constraints can be placed on the blend-source 

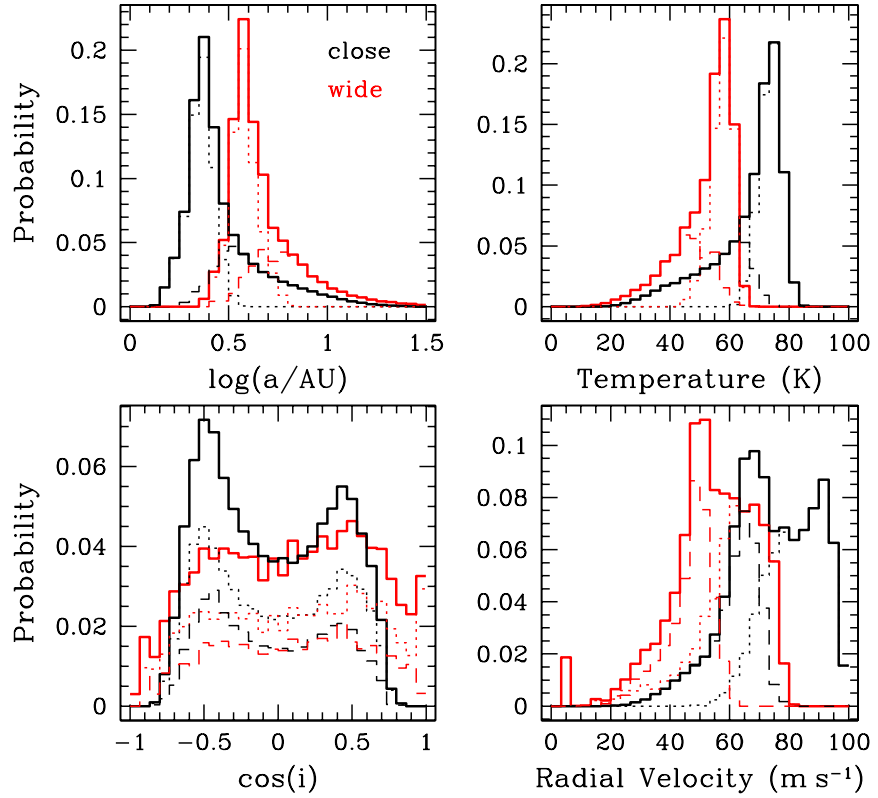

Figure 10. Probability distributions of planetary parameters (semimajor axis $a$, equilibrium temperature, cosine of the inclination, and amplitude of the $\mathrm{RV}$ of the lens star) from MCMC realizations assuming circular orbital motion. Histograms in black and red represent the close-binary and widebinary solutions, respectively. Dotted and dashed histograms represent the two degenerate solutions for each MCMC realization discussed in the Appendix.

separation using the arguments of Section 3.4. These are somewhat more complicated and depend on the blend-source relative parallax, so we do not consider the general case (which would only be of interest to further reduce the already very low probability of a random interloper) but restrict attention to companions of the source and lens. We begin with the simpler source-companion case.

\subsubsection{Blend As A Source Companion}

As we reported in Section 3.4, there were two HST measurements of the astrometric offset between the $V$ and $I$ light centroids, dating from 0.09 and 0.84 years after peak, respectively. In that section, we examined the implications of these measurements under the hypothesis that the blend is the lens. We therefore ignored the first measurement because the lenssource separation at that epoch is much better constrained by the microlensing event itself than by the astrometric measurement. However, as we now examine the hypothesis that the blend is a companion to the source, both epochs must be considered equally. Most of the weight $(86 \%)$ comes from the second observation, partly not because the astrometric errors are slightly smaller, but mainly because the blend contributes about twice the fractional light, which itself reduces the error on the inferred separation by a factor of 2 . Under this hypothesis, we find a bestfit source-companion separation of 5 mas, with a companion P.A. (north through east) of $280^{\circ}$. The (isotropic) error is 3 mas. Approximating the companion-source relative motion as rectilinear, this measurement strictly applies to an epoch 0.73 years after the event, but of course the intrinsic source-companion relative motion must be very small compared to the errors in this measurement.

There would be nothing unusual about such a sourcecompanion projected separation, roughly $40 \pm 25$ AU in physical units. Indeed, the local G-star binary distribution function peaks close to this value (Duquennoy \& Mayor 1991).
The separation derived is also marginally consistent with the companion generating a xallarap signal that mimics the parallax signal in our dominant interpretation. The semimajor axis of the orbit would have to be about $0.8 \mathrm{AU}$ to mimic the $1 \mathrm{yr}$ period of the Earth, which corresponds to a maximum angular separation of about $100 \mu \mathrm{as}$, which is compatible with the astrometric measurements at the $1.6 \sigma$ level.

Another potential constraint comes from comparing the color difference with the magnitude difference of the source and blend. We find that the source is about $0.5 \pm 0.5 \mathrm{mag}$ too bright to be on the same main sequence. However, first, this is only a $1 \sigma$ difference, which is not significant. Second, both the sign and magnitude of the difference are compatible with the source being a slightly evolved turnoff star, which is consistent with its color.

The only present evidence against the source-companion hypothesis is that the astrometric offset between $V$ and I HST images changes between the two epochs, and that the direction and amplitude of this change is consistent with other evidence of the proper motion of the lens. Since this is only a $P=1.7 \%$ effect, it cannot be regarded as conclusive. However, additional HST observations at a later epoch could definitively confirm or rule out this hypothesis.

\subsubsection{Blend As A Lens Companion}

A similar, but somewhat more complicated, line of reasoning essentially rules out the hypothesis that the blend is a companion to the lens, at least if the lens is luminous. The primary difference is that the event itself places very strong lower limits on how close a companion can be to the lens.

A companion with separation (in units of $\left.\theta_{\mathrm{E}}\right) d \gg 1$ induces a Chang \& Refsdal (1979) caustic, which is fully characterized by the gravitational shear $\gamma=q / d^{2}$. We find that the light-curve distortions induced by this shear would be easily noticed unless $\gamma<0.0035$, that is,

$$
\gamma=\frac{q_{c}}{d_{c}^{2}}=\frac{q_{c} \theta_{\mathrm{E}}^{2}}{\theta_{c}^{2}}<0.0035,
$$

where $q_{c}=M_{c} / M$ is the ratio of the companion mass to the lens mass and $d_{c}=\theta_{c} / \theta_{\mathrm{E}}$ is the ratio of the lens-companion separation to the Einstein radius. Equivalently,

$$
\theta_{c}>19\left(\frac{q_{c}}{1.3}\right)^{1 / 2} \theta_{\mathrm{E}}
$$

Here, we have normalized $q_{c}$ to the minimum mass ratio required for the companion to dominate the light assuming that both are main-sequence stars. (We will also consider completely dark lenses below).

We now show that Equation (30) is inconsistent with the astrometric data. If a lens companion is assumed to generate the blend light, then essentially the same line of reasoning given in Section 3.7.1 implies that 0.73 years after the event, this companion lies 5 mas from the source, at a P.A. of $280^{\circ}$ and with an isotropic error of 3 mas. The one wrinkle is that we should now take account of the relative-parallax term in Equation (15), whereas this was identically zero (and so was ignored) for the source-companion case. However, this term is only about $1.8 \pi_{\text {rel }}$ and hence is quite small compared to the measurement errors for typical $\pi_{\text {rel }} \lesssim 0.2$ mas. We will therefore ignore this term in the interest of simplicity, except when we explicitly consider the case of large $\pi_{\text {rel }}$ further below. 


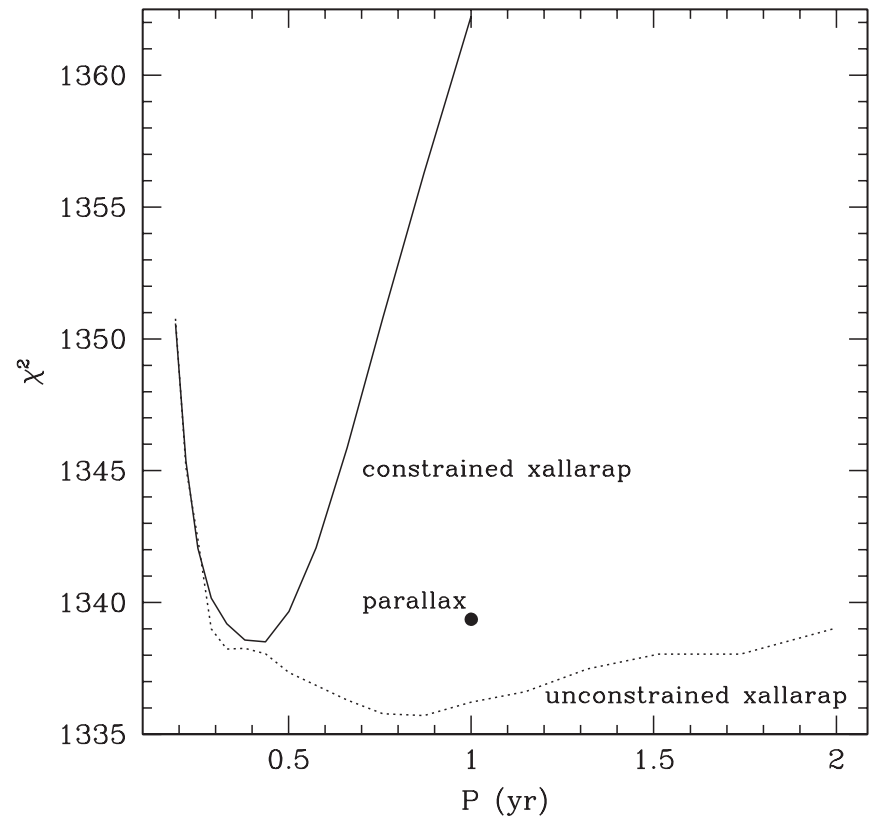

Figure 11. $\chi^{2}$ distributions for best-fit xallarap solutions at fixed binary-source orbital periods $P$. The solid and dotted lines represent xallarap fits with and without dynamical constraints described in Section 3.8. The best-fit parallax solution is shown as a filled dot at a period of one year. All of the fits shown in this figure assume no planetary orbital motion.

Of course, the lens itself moves during this interval. From the parallax measurement alone (i.e., without attributing the $V / I$ astrometric displacement to lens motion), it is known that the lens moves in the same general direction, that is, with the P.A. roughly $210^{\circ}$. In assessing the amplitude of this motion, we consider only the constraints from finite-source effects (and ignore the astrometric displacement). These constraints yield a hard lower limit on $\theta_{\mathrm{E}}$ (from lack of pronounced finite-source effects) of $\theta_{\mathrm{E}}>0.6$ mas, which corresponds to a proper motion

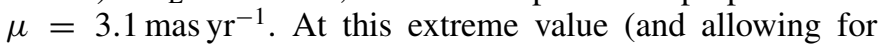
$2 \sigma$ uncertainty in the direction of lens motion as well in the measurement of the companion position), the maximum lenscompanion separation is 11.4 mas (i.e., $19 \theta_{\mathrm{E}}$ ), which is just ruled out by Equation (30). At larger $\theta_{\mathrm{E}}$, the lens-companion scenario is excluded more robustly. For example, in the limit of large $\theta_{\mathrm{E}}$, we have $\theta_{c}=\mu \times 0.73 \mathrm{yr}=\theta_{\mathrm{E}}\left(0.73 \mathrm{yr} / t_{\mathrm{E}}\right)=3.9 \theta_{\mathrm{E}}$, which is clearly ruled out by Equation (30).

Then we note that any scenario involving values of $\pi_{\text {rel }}$ that are large enough that they cannot be ignored in this analysis $\left(\pi_{\text {rel }} \gtrsim\right.$ 0.5 mas) must also have very large $\theta_{\mathrm{E}}=\pi_{\mathrm{rel}} / \pi_{\mathrm{E}} \gtrsim 1$ mas, a regime in which the lens-companion is easily excluded.

The one major loophole to this argument is that the lens may be a stellar remnant (white dwarf, neutron star, or black hole), in which case it could be more massive than the companion despite the latter's greater luminosity.

\subsection{Xallarap Effects and Binary Source}

Binary source motion can give rise to distortions of the light curve, called "xallarap" effects. One can always find a set of xallarap parameters to perfectly mimic parallax distortions caused by the Earth's motion (Smith et al. 2003). However, it is a priori unlikely for the binary source to have such parameters, so if the parallax signal is real, one would expect the xallarap fits to converge to the Earth parameters. For simplicity, we assume that the binary source is in circular orbit. We extensively search the

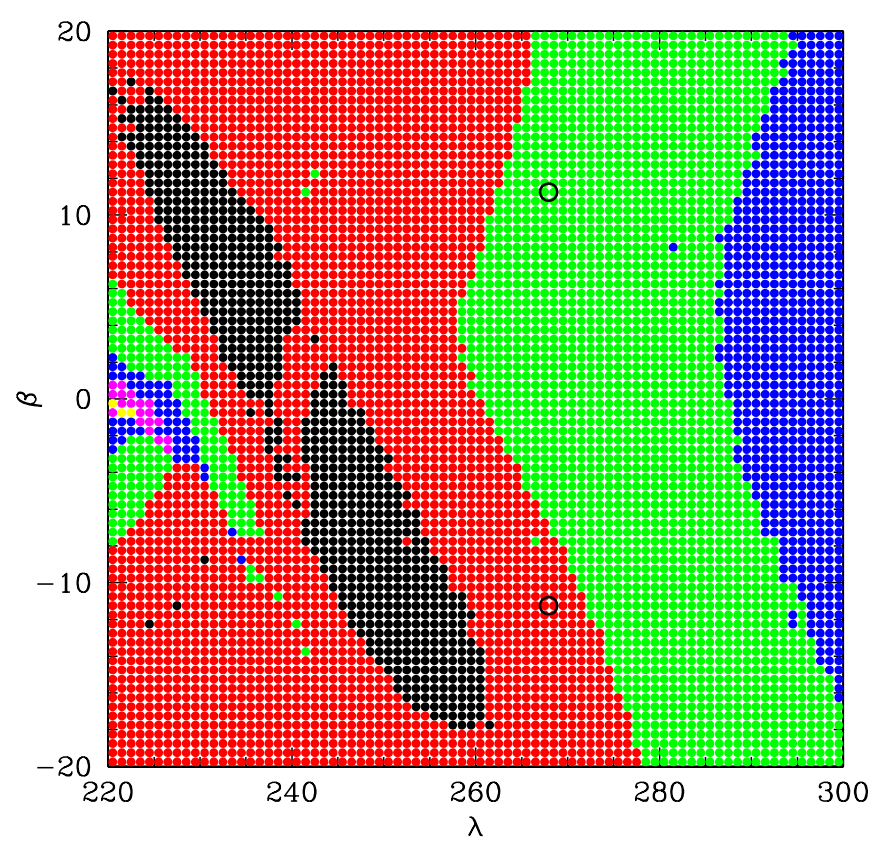

Figure 12. Results of xallarap fits by fixing binary orbital phase $\lambda$ and complement of inclination $\beta$ at period $P=1 \mathrm{yr}$ and $u_{0}>0$. The plot is colorcoded for solutions with $\Delta \chi^{2}$ within 1 (black), 4 (red), 9 (green), 16 (blue), 25 (magenta), and 49 (yellow) of the best fit. The Earth parameters are indicated by black circles. Because of a perfect symmetry $\left(u_{0} \rightarrow-u_{0}\right.$ and $\left.\alpha \rightarrow-\alpha\right)$, the upper black circle represents Earth parameter $\left(\lambda=268^{\circ}, \beta=-11^{\circ}\right)$ for the case $u_{0}<0$. Comparison of parallax with xallarap must be made with the better of the two, that is, the lower one.

parameter space on a grid of five xallarap parameters, namely the period of binary motion $P$, the phase $\lambda$ and complement of inclination $\beta$ of the binary orbit, which corresponds to the ecliptic longitude and latitude in the parallax interpretation of the light curve, as well as $\left(\xi_{\mathrm{E}, \mathrm{E}}, \xi_{\mathrm{E}, \mathrm{N}}\right)$, which are the counterparts of $\left(\pi_{\mathrm{E}, \mathrm{E}}, \pi_{\mathrm{E}, \mathrm{N}}\right)$ of the microlens parallax. We take advantage of the two exact degeneracies found by Poindexter et al. (2005) to reduce the range of the parameter search. One exact degeneracy takes $\lambda^{\prime}=\lambda+\pi$ and $\chi_{E}{ }^{\prime}=-\chi_{E}$, while all other parameters remain the same. The other takes $\beta^{\prime}=-\beta, u_{0}{ }^{\prime}=-u_{0}$, and $\xi_{E, N}^{\prime}=-\xi_{E, N}$ (the sign of $\alpha$ should be changed accordingly as well). Therefore, we restrict our search to solutions with positive $u_{0}$ and with $\pi \leqslant \lambda \leqslant 2 \pi$. In modeling xallarap, planetary orbital motion is neglected. In Figure 11, the $\chi^{2}$ distribution for bestfit xallarap solutions as a function of period is displayed in a dotted line, and the xallarap solution with a period of $1 \mathrm{yr}$ has a $\Delta \chi^{2}=0.5$ larger than the best-fit at 0.9 yr. Figure 12 shows that, for the xallarap solutions with a period of $1 \mathrm{yr}$, the best fit has $\Delta \chi^{2}=3.2$ less than the best-fit parallax solution (displayed as a black circle point) and its orbital parameters are close to the ecliptic coordinates of event $\left(\lambda=268^{\circ}, \beta=-11^{\circ}\right)$. Therefore, the overall best-fit xallarap solution has $\Delta \chi^{2}=3.7$ smaller than that of the parallax solution (whose $\chi^{2}$ value is displayed as a filled dot in Figure 11) for 3 extra degrees of freedom (dof), which gives a probability of $30 \%$. The close proximity between the best-fit xallarap parameters and those of the Earth can be regarded as good evidence of the parallax interpretation. The slight preference of xallarap could simply be statistical fluctuation or reflect low-level systematics in the light curve (commonly found in the analysis by Poindexter et al. 2005).

We also devise another test on the plausibility of xallarap. In Section 3.5, we argued that the blend is unlikely to be a random 
interloper unrelated to either the source or the lens. If the source were in a binary, then the blend would naturally be explained as the companion of the source star. Then from the blend's position on the CMD, its mass would be $m_{c} \sim 0.9 M_{\odot}$. By definition, $\xi_{\mathrm{E}}$ is the size of the source's orbit $a_{s}$ in the units of $\hat{r}_{\mathrm{E}}$ (the Einstein radius projected on the source plane):

$$
\xi_{\mathrm{E}}=\frac{a_{s}}{\hat{r}_{\mathrm{E}}}=\frac{a m_{c}}{\left(m_{c}+m_{s}\right) \hat{r}_{\mathrm{E}}},
$$

where $a$ is the semimajor axis of the binary orbit, and $m_{s}$ and $m_{c}$ are the masses of the source and its companion, respectively. Then we apply Kepler's third law:

$$
\left(\frac{P}{\mathrm{yr}}\right)^{2} \frac{m_{c}^{3}}{M_{\odot}\left(m_{c}+m_{s}\right)^{2}}=\left(\frac{\xi_{\mathrm{E}} \hat{r}_{\mathrm{E}}}{\mathrm{AU}}\right)^{3} .
$$

Once the masses of the source and companion are known, the product of $\xi_{\mathrm{E}}$ and $\hat{r}_{\mathrm{E}}$ are determined for a given binary orbital period $P$. And in the present case, $\hat{r}_{\mathrm{E}} / \mathrm{AU}=\theta_{\mathrm{E}} D_{s}=\theta_{*} / \rho D_{s}=$ $4.5 \times 10^{-3} / \rho$. By adopting $m_{s}=1 M_{\odot}, m_{c}=0.9 M_{\odot}$, for each set of $P$ and $\rho$, there is a uniquely determined $\xi_{\mathrm{E}}$ from Equation (32). We then apply this constraint in the xallarap fitting for a series of periods. The minimum $\chi^{2} s$ for each period from the fittings are shown in a solid line in Figure 11 . The bestfit solution has $\Delta \chi^{2} \sim 1.0$ less than the best-fit parallax solution for 2 extra dof. Although as compared to the test described in the previous paragraph, the current test implies a higher probability that the data are explained by parallax (rather than xallarap) effects, it still does not rule out xallarap.

\section{SUMMARY AND FUTURE PROSPECTS}

Our primary interpretation of the OGLE-2005-BLG-071 data assumes that the light-curve distortions are due to parallax rather than xallarap and that the blended light is due to the lens itself rather than a companion to the source. Under these assumptions, the lens is fairly tightly constrained to be a foreground $\mathrm{M}$ dwarf, with mass $M=0.46 \pm 0.04 M_{\odot}$ and distance $D_{l}=3.2 \pm 0.4 \mathrm{kpc}$, which has thick-disk kinematics $\left(v_{\text {LSR }} \sim 103 \mathrm{~km} \mathrm{~s}^{-1}\right)$. As we discuss below, future observations might help to constrain its metallicity. The microlens modeling suffers from a well known wide-close binary degeneracy. The best-fit wide-binary solutions are slightly favored over the close-binary solutions; however, from dynamical constraints on planetary orbital motion, the physically allowed solutions are not distinguishable within $1 \sigma$. For the wide-binary model, we obtain a planet of mass $M_{p}=3.8 \pm 0.4 M_{\text {Jupiter }}$ at projected separation $r_{\perp}=3.6 \pm 0.2 \mathrm{AU}$. The planet then has an equilibrium temperature of about $T=55 \mathrm{~K}$, that is, similar to Neptune. In the degenerate close-binary solutions, the planet is closer to the star and so hotter, and the estimates are: $M_{p}=3.4 \pm 0.4 M_{\text {Jupiter }}$, $r_{\perp}=2.1 \pm 0.1 \mathrm{AU}$, and $T \sim 71 \mathrm{~K}$.

As we have explored in considerable detail, it is possible that one or both of these assumptions is incorrect. However, future astrometric measurements that are made after the lens and source have had a chance to separate will largely resolve both ambiguities. Moreover, such measurements will put much tighter constraints on the metallicity of the lens (assuming that it proves to be the blended light).

First, the astrometric measurements made 0.84 years after the event detected motion suggests that there was still $1.7 \%$ chance that the blend did not move relative to the source. A later measurement that detected this motion at higher confidence would rule out the hypothesis that the blend is a companion to the source. We argued in Section 3.7.1 that the blend could not be a companion to a main-sequence lens. Therefore, the only possibilities that would remain are that the lens is the blend, that the lens is a remnant (e.g., white dwarf), or that the blend is a random interloper (probability is less than $10^{-3}$ ). As we briefly summarize below, a future astrometric measurement could strongly constrain the remnant-lens hypothesis as well.

Of course, it is also possible that future astrometry will reveal that the blend does not move with respect to the source, in which case the blend would be a companion to the source. Thus, either way, these measurements would largely resolve the nature of the blended light.

Second, by identifying the nature of blend, these measurements will largely, but not entirely, resolve the issue of parallax versus xallarap. If the blend proves not to be associated with the source, then any xallarap-inducing companion would have to be considerably less luminous and so (unless it were a neutron star) less massive than the $m_{c}=0.9 M_{\odot}$ that we assumed in evaluating Equation (32). Moreover, stronger constraints on $\hat{r}_{\mathrm{E}}$ (right-hand side of Equation (32)) would be available from the astrometric measurements. Hence, the xallarap option would be either excluded or very strongly constrained by this test.

On the other hand, if the blend were confirmed to be a source companion, then essentially all higher order constraints on the nature of the lens would disappear. The parallax "measurement" would then very plausibly be explained by xallarap, while the "extra information" about $\theta_{\mathrm{E}}$ that is presently assumed to come from the blend proper-motion measurement would likewise evaporate.

These considerations strongly argue for making a future high-precision astrometric measurement. Recall that in the HST measurements reported in Section 3.5, the source and blend were not separately resolved: the relative motion was inferred from the offset between the $V$ and $I$ centroids, which are displaced because the source and blend have different colors. Due to its well controlled PSF, HST is capable of detecting the broadening of the PSF even if the separation of the lens and source is a fraction of the FWHM. Assuming that the proper

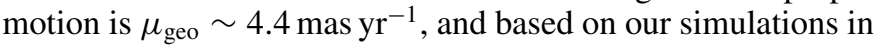
Section 3.5 , such broadening would be confidently detectable about 5 years after the event (see also Bennett et al. 2007 for analytic PSF broadening estimates). Ten years after the event, the net displacement would be $\sim 40$ mas. This compares to a diffraction-limited FWHM of 40 mas for the $H$ band on a ground-based $10 \mathrm{~m}$ telescope and would therefore enable full resolution. The $I-H$ color of the source is extremely well determined $(0.01 \mathrm{mag})$ from simultaneous $I$ and $H$ data taken during the event from the CTIO/SMARTS $1.3 \mathrm{~m}$ in Chile. Hence, the flux allocation of the partially or fully resolved blend and source stars would be known. The direct detection of a partially or fully resolved lens will provide precise photometric and astrometry measurements (see Kozłowski et al. 2007 for one such example), which will enable much tighter constraints on the mass, distance, and projected velocity of the lens. It also opens up the possibility of determining the metallicity of the host star by taking into account both nonphotometric and photometric constraints. If, as indicated by the projected velocity measurement, it is a thick-disk star, then it will be one of the few such stars found to harbor a planet (Haywood 2008).

As remarked above, a definitive detection of the blend's proper motion would still leave open the possibility that it was 
a companion to the lens, and not the lens itself. In this case, the lens would have to be a remnant. Without going into detail, the astrometric measurement would simultaneously improve the blend color measurement as well as giving a proper-motion estimate (albeit with large errors because the blend-source offset at the peak of the event would then not be known). It could then be asked whether the parallax, proper-motion, and photometric data could be consistently explained by any combination of remnant lens and main-sequence companion. This analysis would depend critically on the values of the measurements, so we do not explore it further here. We simply note that this scenario could also be strongly constrained by future astrometry.

\section{DISCUSSION}

With the measurements presented here, and the precision with which these measurements allow us to determine the properties of the planet OGLE-2005-BLG-071Lb and its host, it is now possible to place this system in the context of similar planetary systems discovered by RV surveys. Of course, the kind of information that can be inferred about the planetary systems discovered via RV differs somewhat from that presented here. For example, for planets discovered via RV, it is generally only possible to infer a lower limit to the planet mass, unless the planets happen to transit or produce a detectable astrometric signal. Mutatis mutandis, for planets discovered via microlensing, it is generally only possible to measure the projected separation at the time of the event, even in the case for which the microlensing mass degeneracy is broken as it is here (although see Gaudi et al. 2008).

With these caveats in mind, we can compare the properties of OGLE-2005-BLG-071Lb and its host star with similar RV systems. It is interesting to note that the fractional uncertainties in the host mass and distance of OGLE-2005-BLG-071Lb are comparable to those of some of the systems listed in Table 3.

OGLE-2005-BLG-071Lb is one of the only eight Jovianmass $\left(0.2 M_{\text {Jupiter }}<M_{p}<13 M_{\text {Jupiter }}\right)$ planets that have been detected orbiting $\mathrm{M}$ dwarf hosts (i.e., $M_{*}<0.55 M_{\odot}$; Marcy et al. 1998, 2001; Delfosse et al. 1998; Butler et al. 2006; Johnson et al. 2007b; Bailey et al. 2009). Table 3 summarizes the planetary and host-star properties of the known M dwarf/Jovianmass planetary systems. OGLE-2005-BLG-071Lb is likely the most massive known planet orbiting an M dwarf.

As suggested by the small number of systems listed in Table 3 , and shown quantitatively by several recent studies, the frequency of relatively short-period $P \lesssim 2000$ days, Jupitermass companions to $\mathrm{M}$ dwarfs appears to be $\sim 3-5$ times lower than such companions to FGK dwarfs (Butler et al. 2006; Endl et al. 2006; Johnson et al. 2007b; Cumming et al. 2008). This paucity, which has been shown to be statistically significant, is expected in the core-accretion model of planet formation, which generally predicts that Jovian companions to M dwarfs should be rare, since for lower mass stars, the dynamical time at the sites of planet formation is longer, whereas the amount of raw material available for planet formation is smaller (Laughlin et al. 2004; Ida \& Lin 2005; Kennedy \& Kenyon 2008, but see Kornet et al. 2006). Thus, these planets typically do not reach sufficient mass to accrete a massive gaseous envelope over the lifetime of the disk. Consequently, such models also predict that in the outer regions of their planetary systems, lower mass stars should host a much larger population of "failed Jupiters," cores of mass $\lesssim 10 M_{\oplus}$ (Laughlin et al. 2004; Ida \& Lin 2005). Such a population was indeed identified based on two microlensing planet discoveries (Beaulieu et al. 2006; Gould et al. 2006).

Our detection of a $\sim 4 M_{\text {Jupiter }}$ companion to an M dwarf may therefore present a difficulty for the core-accretion scenario. While we do not have a constraint on the metallicity of the host, the fact that it is likely a member of the thick disk suggests that its metallicity may be subsolar. If so, this would pose an additional difficulty for the core-accretion scenario, which also predicts that massive planets should be rarer around metal-poor stars (Ida \& Lin 2004), as has been demonstrated observationally (Santos et al. 2004; Fischer \& Valenti 2005). This might imply that a different mechanism is responsible for planet formation in the OGLE-2005-BLG-071L system, such as the gravitational instability mechanism (Boss 2002, 2006).

One way to escape these potential difficulties is if the host lens is actually a stellar remnant, such as a white dwarf. The progenitors of remnants are generally more massive stars, which are both predicted (Ida \& Lin 2005; Kennedy \& Kenyon 2008) and observed (Johnson et al. 2007a, 2007b) to have a higher incidence of massive planets. As we discussed above, future astrometric measurements could constrain both the lowmetallicity and remnant-lens hypotheses. These measurements are therefore critical.

Although it is difficult to draw robust conclusions from a single system, there are now four published detections of Jovian-mass planetary companions with microlensing (Bond et al. 2004; Gaudi et al. 2008), and several additional such planets have been detected that are currently being analyzed. It is therefore reasonable to expect several detections per year (Gould 2009), and thus it will soon be possible to use microlensing to constrain the frequency of massive planetary companions. These constraints are complementary to those from RV, since the microlensing detection method is less biased with respect to host star mass (Gould 2000a), and furthermore probes a different region of parameter space, namely cool planets beyond the snow line with equilibrium temperatures similar to the giant planets in our solar system (see, e.g., Gould et al. 2007; Gould 2009).

We thank M. Pinsonneault and D. An for providing us their unpublished isochrones. S.D. wishes to thank D. Will of Ohio State astronomy department for setting up and maintaining the Condor system, which greatly facilitates the computations for this work. S.D. is grateful to O. Pejcha and D. Heyrovsky for interesting discussions on limb-darkening. Based on observations with the NASA/ESA HST obtained at the Space Telescope Science Institute, which is operated by the Association of Universities for Research in Astronomy, Incorporated, under NASA contract NAS5-26555. Support for this work was provided by NASA through grant HST-GO-10707.01-A from STScI. S.D. and A.G. were supported in part by grant AST 042758 from the NSF. S.D., A.G., D.D., and R.P. acknowledge support by NASA grant NNG04GL51G. A.G. thanks IAP, CNRS for its support. Support for OGLE project was provided by Polish MNiSW grant N20303032/4275. B.G.P. was supported by the grant (KRF-2006-311-C00072) from Korea Research Foundation. H.C. was supported by the Science Research Center from Korea Science and Engineering Foundation. The MOA project is supported by Ministry of Education, Culture, Sports, Science and Technology (MEXT) of Japan, Grant-in-Aid for Specially Promoted Research No. 14002006. J.P.B., P.F., A.C., C.C., S.B., J.B.M. acknowledge the financial support of ANR HOLMES. K.H.C.'s work was performed under the auspices of 
Table 3

Jovian-mass Companions to M Dwarfs $\left(M_{*}<0.55 M_{\odot}\right)$

\begin{tabular}{|c|c|c|c|c|c|c|c|}
\hline Name & $\begin{array}{c}M_{*} \\
\left(M_{\odot}\right)\end{array}$ & Metallicity & $\begin{array}{l}\text { Dist. } \\
\text { (pc) }\end{array}$ & $\begin{array}{c}M_{p} \\
\left(M_{\text {Jup }}\right)\end{array}$ & $\begin{array}{c}P \\
\text { (days) }\end{array}$ & $\begin{array}{c}a \\
(\mathrm{AU})\end{array}$ & Ref. \\
\hline GJ 876c & $\begin{array}{c}0.32 \\
\pm 0.03\end{array}$ & $\begin{array}{l}-0.12 \\
\pm 0.12\end{array}$ & $\begin{array}{c}4.660 \\
\pm 0.004\end{array}$ & $\begin{array}{c}0.6- \\
0.8\end{array}$ & $\begin{array}{l}30.340 \\
\pm 0.013\end{array}$ & 0.13030 & $1,2,3$ \\
\hline GJ 876b & $\ldots$ & $\ldots$ & $\ldots$ & $\begin{array}{c}1.9- \\
2.5\end{array}$ & $\begin{array}{l}60.940 \\
\pm 0.013\end{array}$ & 0.20783 & $\ldots$ \\
\hline GJ 849b & $\begin{array}{c}0.49 \\
\pm 0.05\end{array}$ & $\begin{array}{l}0.16 \\
\pm 0.2\end{array}$ & $\begin{array}{c}8.8 \\
\pm 0.2\end{array}$ & $0.82 / \sin i$ & $\begin{array}{l}1890 \\
\pm 130\end{array}$ & 2.35 & 4 \\
\hline GJ 317b & $\begin{array}{c}0.24 \\
\pm 0.04\end{array}$ & $\begin{array}{l}-0.23 \\
\pm 0.2\end{array}$ & $\begin{array}{c}9.2 \\
\pm 1.7\end{array}$ & $1.2 / \sin i$ & $\begin{array}{c}692.9 \\
\pm 4\end{array}$ & 0.95 & 5 \\
\hline GJ 832b & $\begin{array}{c}0.45 \\
\pm 0.05\end{array}$ & $\begin{array}{l}\sim-0.7 \\
/-0.3\end{array}$ & $\sim 4.93$ & $0.64 / \sin i$ & $\begin{array}{l}3416 \\
\pm 131\end{array}$ & $\begin{array}{c}3.4 \\
\pm 0.4\end{array}$ & 6 \\
\hline $\begin{array}{l}\text { OGLE-2006 } \\
\text {-BLG-109Lb }\end{array}$ & $\begin{array}{c}0.50 \\
\pm 0.05\end{array}$ & $\begin{array}{l}? \\
?\end{array}$ & $\begin{array}{l}1490 \\
\pm 130\end{array}$ & $\begin{array}{c}0.71 \\
\pm 0.08\end{array}$ & $\begin{array}{l}1830 \\
\pm 370\end{array}$ & $\begin{array}{c}2.3 \\
\pm 0.2\end{array}$ & 7 \\
\hline $\begin{array}{l}\text { OGLE-2006 } \\
\text {-BLG-109Lc }\end{array}$ & $\ldots$ & $\ldots$ & $\ldots$ & $\begin{array}{c}0.27 \\
\pm 0.03\end{array}$ & $\begin{array}{l}5100 \\
\pm 730\end{array}$ & $\begin{array}{c}4.6 \\
\pm 0.5\end{array}$ & $\ldots$ \\
\hline $\begin{array}{l}\text { OGLE-2005 } \\
\text {-BLG-071Lb }\end{array}$ & $\begin{array}{c}0.46 \\
\pm 0.04\end{array}$ & Subsolar? ${ }^{\mathrm{a}}$ & $\begin{array}{l}3300 \\
\pm 300\end{array}$ & $\begin{array}{l}3.8^{\mathrm{b}} \\
\pm 0.4\end{array}$ & $\ldots$ & $\begin{array}{l}3.6^{\mathrm{b}, \mathrm{c}} \\
\pm 0.2\end{array}$ & $\begin{array}{l}\text { This } \\
\text { paper }\end{array}$ \\
\hline
\end{tabular}

Notes.

${ }^{a}$ While the metallicity of the OGLE-2005-BLG-071Lb host star is not directly constrained by our data, its kinematics indicate that it is likely a member of the metal-poor thick disk.

b We give the planet mass and projected separation for the wide solution, which is favored by $\Delta \chi^{2}=2.1$. The second, close solution has $M_{p}=3.4 \pm 0.3 M_{\text {Jupiter }}$ and $r_{\perp}=2.1 \pm 0.1 \mathrm{AU}$.

${ }^{c}$ We give the projected separation between the host and planet at the time of event, which is the orbital parameter most directly constrained by our observations. However, assuming a circular orbit, we infer that the semimajor axis is likely only $\sim 10 \%-20 \%$ larger ( $a$ (wide) $\sim 4.1 \mathrm{AU}, a$ (close) $\sim 2.5 \mathrm{AU}$ ).

References. (1) Rivera et al. 2005; (2) Bean et al. 2006; (3) Benedict et al. 2002; (4) Butler et al. 2006; (5) Johnson et al. $2007 \mathrm{~b}$ (6) Bailey et al. 2009 (7) Gaudi et al. 2008.

the U.S. Department of Energy by Lawrence Livermore National Laboratory under Contract DE-AC52-07NA27344. This work was supported in part by an allocation of computing time from the Ohio Supercomputer Center.

\section{APPENDIX}

\section{EXTRACTING ORBITAL PARAMETERS FOR A CIRCULAR PLANETARY ORBIT}

OGLE-2005-BLG-071 is the first planetary microlensing event for which the effects of planetary orbital motion in the light curve have been fully analyzed. The distortions of the light curve due to the orbital motion are modeled by $\omega$ and $\dot{b}$ as discussed in Section 3.2. In addition, the lens mass $M$ and distance $D_{l}$ are determined, so we can directly convert the microlens lightcurve parameters that are normalized to the Einstein radius to physical parameters. In this section, we show that under the assumption of a circular planetary orbit, the planetary orbital parameters can be deduced from the light-curve parameters. Let $r_{\perp}=D_{l} \theta_{\mathrm{E}} d$ be the projected star-planet separation and let $r_{\perp} \boldsymbol{\gamma}$ be the instantaneous planet velocity in the plane of the sky, that is, $r_{\perp} \gamma_{\perp}=r_{\perp} \omega$ is the velocity perpendicular to this axis and $r_{\perp} \gamma_{\|}=r_{\perp} \dot{d} / d$ is the velocity parallel to this axis. Let $a$ be the semimajor axis and define the $\hat{\boldsymbol{\imath}}, \hat{\boldsymbol{j}}, \hat{\boldsymbol{k}}$ directions as the instantaneous star planet axis on the sky plane, the direction into the sky, and $\hat{\boldsymbol{k}}=\hat{\boldsymbol{\imath}} \times \hat{\boldsymbol{j}}$. Then the instantaneous velocity of the planet is

$$
\mathbf{v}=\sqrt{\frac{G M}{a}}[\cos \theta \hat{\boldsymbol{k}}+\sin \theta(\cos \phi \hat{\boldsymbol{\imath}}-\sin \phi \hat{\boldsymbol{j}})]
$$

where $\phi$ is the angle between the star-planet-observer (i.e., $\left.r_{\perp}=a \sin \phi\right)$ and $\theta$ is the angle of the velocity relative to the $\hat{\boldsymbol{k}}$ direction on the plane that is perpendicular to the planet-star axis. We thus obtain

$$
\gamma_{\perp}=\sqrt{\frac{G M}{a^{3}}} \frac{\cos \theta}{\sin \phi}, \quad \gamma_{\|}=\sqrt{\frac{G M}{a^{3}}} \sin \theta \cot \phi .
$$

To facilitate the derivation, we define

$$
A \equiv \frac{\gamma_{\|}}{\gamma_{\perp}}=-\tan \theta \cos \phi, \quad B \equiv \frac{r_{\perp}^{3} \gamma_{\perp}^{2}}{G M}=\cos ^{2} \theta \sin \phi,
$$

which yield as an equation for $\sin \phi$ :

$$
B=F(\sin \phi) ; \quad F(x)=\frac{x\left(1-x^{2}\right)}{A^{2}+1-x^{2}} .
$$

Note that $F^{\prime}(\sin \phi)=0$ when $\sin ^{2} \phi_{*}=(3 / 2) A^{2}+1-$ $|A| \sqrt{(9 / 4) A^{2}+2}$. So Equation (A4) has two degenerate solutions when $B<F\left(\sin \phi_{*}\right)$ and has no solutions when $B>F\left(\sin \phi_{*}\right)$. Subsequently, one obtains

$$
a=\frac{r_{\perp}}{\sin \phi}, \quad \cos i=-\sin \phi \cos \theta, \quad K=\sqrt{\frac{G M}{a}} q \sin i,
$$

where $i$ is the inclination and $K$ is the amplitude of the RV.

The Jacobian matrix used to transform from $P\left(r_{\perp}, \gamma_{\perp}, \gamma_{\|}\right)$to $P(a, \phi, \theta)$ is given below:

$$
\frac{\partial\left(r_{\perp}, \gamma_{\perp}, \gamma_{\|}\right)}{\partial(a, \phi, \theta)}=\frac{G M}{a^{3}}\left|\begin{array}{ccc}
\sin \phi & a \cos \phi & 0 \\
-\frac{3}{2 a} \frac{\cos \theta}{\sin \phi} & -\frac{\cos \theta \cos \phi}{\sin ^{2} \phi} & -\frac{\sin \theta}{\sin \phi} \\
-\frac{3}{2 a} \sin \theta \cot \phi & -\frac{\sin \theta}{\sin ^{2} \phi} & \cos \theta \cot \phi
\end{array}\right|
$$


No. 2, 2009

$$
=\frac{G M}{a^{3}} \cot ^{2} \phi\left(\frac{1}{2}-\sin ^{2} \theta \tan ^{2} \phi\right) \text {. }
$$

Then for an arbitrary function $H(a)$,

$$
\frac{\partial\left(r_{\perp}, \gamma_{\perp}, \gamma_{\|}\right)}{\partial(H(a), \cos \phi, \theta)}=\frac{\partial\left(r_{\perp}, \gamma_{\perp}, \gamma_{\|}\right)}{\partial(a, \phi, \theta)} \times \frac{1}{\sin \phi H^{\prime}(a)},
$$

which, for the special case of a flat distribution, $H(a)=\ln a$, yields

$$
\frac{\partial\left(r_{\perp}, \gamma_{\perp}, \gamma_{\|}\right)}{\partial(\ln (a), \cos \phi, \theta)}=\frac{G M}{r_{\perp}^{2}} \frac{\cos ^{2} \phi}{\sin \phi} \cdot\left(\frac{1}{2}-\sin ^{2} \theta \tan ^{2} \phi\right) .
$$

\section{REFERENCES}

Albrow, M. D., et al. 1999, ApJ, 512, 672

An, J. H. 2005, MNRAS, 356, 1409

An, J. H., et al. 2002, ApJ, 572, 521

Anderson, J., \& King, I. R. 2004, Hubble Space Telescope Advanced Camera

for Surveys Instrument Science Report 04-15

Bailey, J., et al. 2009, ApJ, 690, 743

Bean, J. L., Benedict, G. F., \& Endl, M. 2006, ApJ, 653, L65

Beaulieu, J.-P., et al. 2006, Nature, 439, 437

Benedict, G. F., et al. 2002, ApJ, 581, L115

Bennett, D. P., Anderson, J., \& Gaudi, B. S. 2007, ApJ, 660, 781

Bennett, D. P., et al. 2006, ApJ, 647, L171

Bessell, M. S., \& Brett, J. M. 1988, PASP, 100, 1134

Bond, I. A., et al. 2004, ApJ, 606, L155

Boss, A. P. 2002, ApJ, 567, L149

Boss, A. P. 2006, ApJ, 643, 501

Butler, R. P., et al. 2006, PASP, 118, 1685

Chang, K., \& Refsdal, S. 1979, Nature, 282, 561

Claret, A. 2000, A\&A, 363, 8081

Cumming, A., et al. 2008, PASP, 120, 531

Delfosse, X., et al. 1998, A\&A, 338, L67

Dominik, M. 1999, A\&A, 349, 108

Dong, S., et al. 2006, ApJ, 642, 842

Dong, S., et al. 2008, arXiv:0809.2997

Doran, M., \& Mueller, C. M. 2004, JCAP, 09, 003
Duquennoy, A., \& Mayor, M. 1991, A\&A, 248, 485

Endl, M., et al. 2006, ApJ, 649, 436

Fischer, D. A., \& Valenti, J. 2005, ApJ, 622, 1102

Gaudi, B. S., et al. 2008, in ASP Conf. Ser. 398, Extreme Solar Systems, ed. D. Fischer et al. (San Francisco, CA: ASP), 479

Gaudi, B. S., et al. 2008, Science, 319, 927

Gould, A. 1992, ApJ, 392, 442

Gould, A. 1994, ApJ, 421, 171

Gould, A. 2000a, ApJ, 535, 928

Gould, A. 2000b, ApJ, 542, 785

Gould, A. 2004, ApJ, 606, 319

Gould, A. 2008, ApJ, 681, 1593

Gould, A. 2009, in ASP Conf. Ser. 403, The Variable Universe: A Celebration of Bohdan Paczynski, ed. K. Z. Stanek (San Francisco, CA: ASP), 86

Gould, A., Gaudi, B. S., \& Bennett, D. P. 2007, NASA/NSF Exoplanet Task Force White Paper (arXiv:0704.0767)

Gould, A., Miralda Escude, J., \& Bahcall, J. N. 1994, ApJ, 423, L105

Gould, A., et al. 2006, ApJ, 644, L37

Kervella, P., Thévenin, F., Di Folco, E., \& Ségransan, D. 2004, A\&A, 426, 297

Kozłowski, S., Woźniak, P. R., Mao, S., \& Wood, A. 2007, ApJ, 671, 420

Kubas, D., et al. 2008, A\&A, 483, 317

Haywood, M. 2008, A\&A, 482, 673

Ida, S., \& Lin, D. N. C. 2004, ApJ, 616, 567

Ida, S., \& Lin, D. N. C. 2005, ApJ, 626, 1045

Johnson, J. A., et al. 2007a, ApJ, 665, 785

Johnson, J. A., et al. 2007b, ApJ, 670, 833

Kennedy, G. M., \& Kenyon, S. J. 2008, ApJ, 673, 502

Kornet, K., Wolf, S., \& Różyczka, M. 2006, A\&A, 458, 661

Laughlin, G., Bodenheimer, P., \& Adams, F. C. 2004, ApJ, 612, L73

Lejeune, T., Cuisinier, F., \& Buser, R. 1997, A\&AS, 125, 229

Lejeune, T., Cuisinier, F., \& Buser, R. 1998, A\&AS, 130, 65

Manfroid, J. 1995, A\&AS, 113, 587

Marcy, G. W., et al. 1998, ApJ, 505, L147

Marcy, G. W., et al. 2001, ApJ, 556, 296

Nishiyama, S., et al. 2005, ApJ, 621, L105

Pejcha, O., \& Heyrovsky, D. 2009, ApJ, 690, 1772

Poindexter, S., et al. 2005, ApJ, 633, 914

Rivera, E. J., et al. 2005, ApJ, 634, 625

Santos, N. C., Israelian, G., \& Mayor, M. 2004, A\&A, 415, 1153

Smith, M. C., Mao, S., \& Paczyński, B. 2003, MNRAS, 339, 925

Udalski, A., et al. 2005, ApJ, 628, L109 (Paper I)

Walker, M. A. 1995, ApJ, 453, 37

Wozniak, P. R. 2000, Acta Astron., 50, 421

Yoo, J., et al. 2004, ApJ, 603, 139 